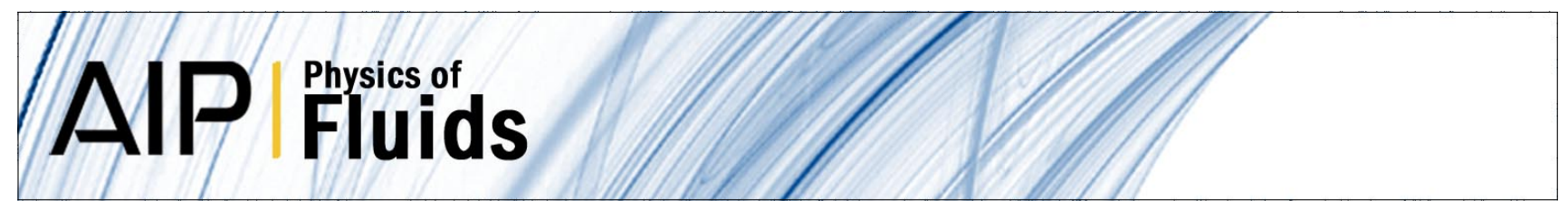

\title{
Compressible laminar streaks with wall suction
}

Pierre Ricco, Daniel Shah, and Peter D. Hicks

Citation: Phys. Fluids 25, 054110 (2013); doi: 10.1063/1.4807066

View online: http://dx.doi.org/10.1063/1.4807066

View Table of Contents: http://pof.aip.org/resource/1/PHFLE6/v25/i5

Published by the American Institute of Physics.

\section{Additional information on Phys. Fluids}

Journal Homepage: http://pof.aip.org/

Journal Information: http://pof.aip.org/about/about_the_journal

Top downloads: http://pof.aip.org/features/most_downloaded

Information for Authors: http://pof.aip.org/authors

\section{ADVERTISEMENT}

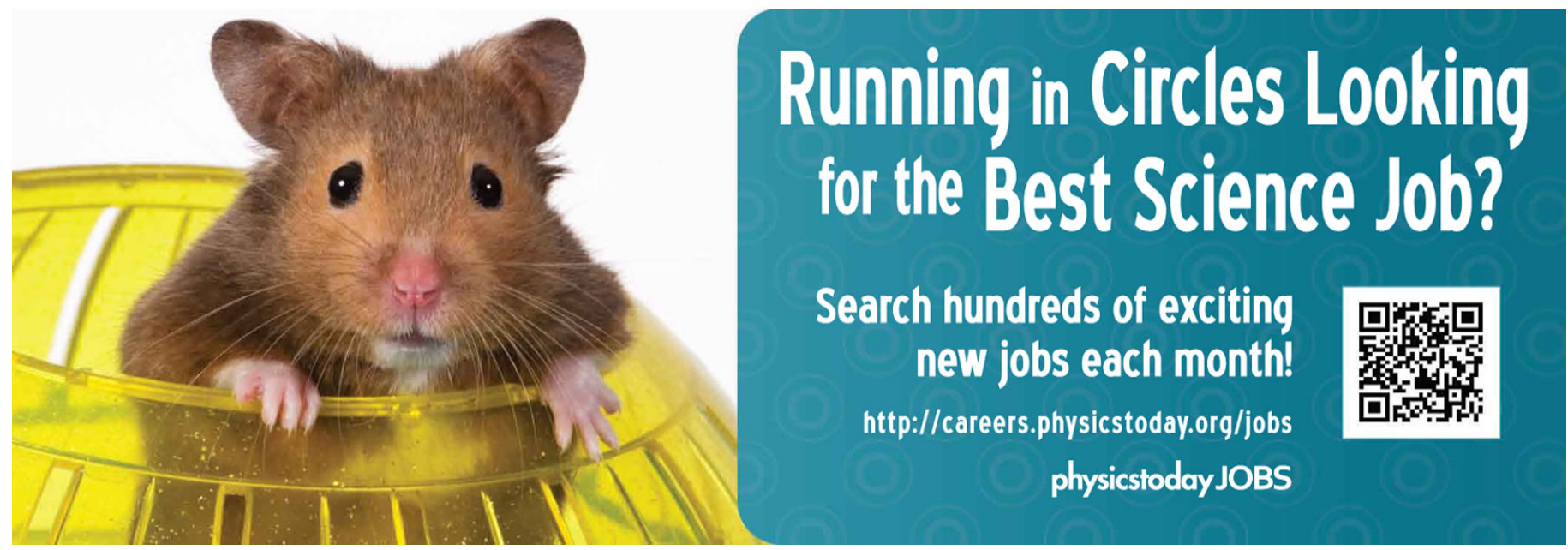




\title{
Compressible laminar streaks with wall suction
}

\author{
Pierre Ricco, ${ }^{1}$ Daniel Shah, ${ }^{2}$ and Peter D. Hicks ${ }^{3}$ \\ ${ }^{1}$ Department of Mechanical Engineering, The University of Sheffield, Mappin Street, \\ Sheffield S1 3JD, United Kingdom \\ ${ }^{2}$ Department of Mechanical Engineering, Imperial College London, South Kensington, \\ London SW7 2AZ, United Kingdom \\ ${ }^{3}$ School of Engineering, Fraser Noble Building, King's College, The University of Aberdeen, \\ Aberdeen AB24 3UE, United Kingdom
}

(Received 22 October 2012; accepted 22 April 2013; published online 29 May 2013)

The response of a compressible laminar boundary layer subject to free-stream vortical disturbances and steady mean-flow wall suction is studied. The theoretical frameworks of Leib et al. [J. Fluid Mech. 380, 169-203 (1999)] and Ricco and Wu [J. Fluid Mech. 587, 97-138 (2007)], based on the linearized unsteady boundaryregion equations, are adopted to study the influence of suction on the kinematic and thermal streaks arising through the interaction between the free-stream vortical perturbations and the boundary layer. In the asymptotic limit of small spanwise wavelength compared with the boundary layer thickness, i.e., when the disturbance flow is conveniently described by the steady compressible boundary region equations, the effect of suction is mild on the velocity fluctuations and negligible on the temperature fluctuations. When the spanwise wavelength is comparable with the boundary layer thickness, small suction values intensify the supersonic streaks, while higher transpiration levels always stabilize the disturbances at all Mach numbers. At larger spanwise wavelengths, very small amplitudes of wall transpiration have a dramatic stabilizing effect on all boundary layer fluctuations, which can take the form of transiently growing thermal streaks, large amplitude streamwise oscillations, or oblique exponentially growing Tollmien-Schlichting waves, depending on the Mach number and the wavelengths. The range of wavenumbers for which the exponential growth occurs becomes narrower and the location of instability is significantly shifted downstream by mild suction, indicating that wall transpiration can be a suitable vehicle for delaying transition when the laminar breakdown is promoted by these unstable disturbances. The typical streamwise wavelength of these disturbances is instead not influenced by suction, and asymptotic triple deck theory predicts the strong changes in growth rate and the very mild modifications in streamwise wavenumber in the limit of larger downstream distance and small spanwise wavenumber. (๐ 2013 AIP Publishing LLC. [http://dx.doi.org/10.1063/1.4807066]

\section{INTRODUCTION}

The transition from laminar to turbulent flow within a boundary layer is of critical importance in understanding the behaviour of many high-speed fluid flows around aerofoils and within turbomachinery. Transition can be initiated through a variety of mechanisms, such as the interactions of the boundary layer with surface roughness ${ }^{26}$ or free-stream disturbances, ${ }^{10}$ which may be in the form of vortical, acoustic, and temperature fluctuations. Upstream of the transition point, the interplay between free-stream disturbances and the boundary layer may lead to disturbances which, if they persist and grow, can become unstable, ultimately triggering transition to turbulence. The mechanism through which laminar-turbulent transition occurs and the location of the transition point depend upon the free-stream disturbance intensity $T u$. Generally, the transition point moves upstream as $T u$ increase. A notable exception is the case of swept-wing laminar boundary layers for which a higher $T u$ leads to a lower transition Reynolds number because travelling waves are enhanced in 
lieu of stationary cross-flow vortices, which instead cause transition explosively through secondary instability when $T u$ is low. ${ }^{8}$

Over a flat smooth plate, the growth of viscous Tollmien-Schlichting (TS) waves is responsible for transition at low levels $(T u<0.1 \%)$. With more intense free-stream disturbances $(T u>1 \%)$, transition may occur without TS waves, instead involving low-frequency streamwise-elongated streaks. These streaks were first observed experimentally by Dryden ${ }^{9}$ and Taylor, ${ }^{37}$ who called them breathing modes. The disturbances have been more recently associated with Klebanoff ${ }^{19}$ and are often referred to as Klebanoff modes. ${ }^{18,38}$ Experiments have shown that the Klebanoff modes experience a substantial downstream growth before undergoing transition and forming localized turbulent spots. ${ }^{25}$ The turbulent spots eventually become larger and combine to generate the fully developed turbulent flow.

The rigorous mathematical description of the pre-transitional Klebanoff modes in the incompressible regime was developed by Leib, Wundrow, and Goldstein ${ }^{21}$ (subsequently referred to as LWG), Wundrow and Goldstein, ${ }^{41} \mathrm{Wu}$ and Choudhari, ${ }^{40}$ and Ricco, ${ }^{29}$ while the extension to the compressible regime was carried out by Ricco and $\mathrm{Wu}^{33}$ (subsequently referred to as $\mathrm{RW}$ ). It is shown in these studies that the precise mathematical specification of the initial conditions near the leading edge and of the non-trivial interplay between the boundary layer disturbances and the free-stream fluctuations is crucial to capture the dynamics of the streaks. Furthermore, LWG showed that the free-stream forcing must be synthesized continuously along the whole streamwise extent and that non-parallel effects must be retained to obtain the correct downstream streak evolution.

An alternative mathematical formulation describing the pre-transitional laminar streaks induced by free-stream vortical disturbances was proposed by Andersson et al. ${ }^{1}$ and Luchini. ${ }^{24}$ It is usually referred to as optimal growth theory. The crucial difference from the frameworks developed by LWG, Wundrow and Goldstein, ${ }^{41}$ and $\mathrm{Wu}$ and Choudhari ${ }^{40}$ is that the free-stream perturbations are not taken into account. Therefore, the initial conditions cannot be derived asymptotically from the external disturbances as in LWG; they are instead found through an iterative adjoint method which maximizes the disturbance growth. Another important difference is that the optimal growth theory predicts the laminar streaks to be steady, while in LWG's theory the streaks are unsteady and their frequency of oscillation is one of the free-stream perturbation as shown by experiments. ${ }^{25}$

Controlling the growth of instabilities within a laminar boundary layer with the aim of either delaying transition or entirely preventing the onset of turbulence is important for reducing the friction drag and increasing the aircraft fuel efficiency. The drag produced by a turbulent boundary layer is much greater than in the laminar case and, therefore, if the proportion of an aerofoil covered by laminar flow can be extended, then a reduction of the overall drag will be achieved. Flow control research has been focusing on the reduction in disturbance growth during the initial flow development as the perturbations are less energetic than when nonlinear effects become relevant or when the flow is fully turbulent. ${ }^{13}$

Wind-tunnel experiments have shown that wall suction can attenuate the growth of streaks. ${ }^{12,42}$ The effect of wall suction on the laminar streaks has also been studied through direct numerical simulations, ${ }^{22}$ optimal growth theory, ${ }^{6,11,43}$ and by Ricco and Dilib, who used LWG's mathematical framework. ${ }^{30}$ For aircraft flight, the use of wall suction as a mechanism for controlling the disturbance growth has also been widely investigated. ${ }^{5,17}$ In the present study, we study the effect of wall suction on the compressible Klebanoff modes generated by free-stream vortical perturbations for the first time, in line with other works on the effect of suction on other pre-transitional disturbances, such as TS waves ${ }^{4}$ and Görtler vortices. ${ }^{2,3}$ We build on the incompressible flow study of Ricco and Dilib $^{30}$ and use the theoretical framework of compressible boundary layer streaks developed by RW. Section II describes the mathematical formulation, while Sec. III presents the results for different streak regimes, distinguished by the scaled spanwise wavenumber $\kappa$. A summary is contained in Sec. IV.

\section{MATHEMATICAL FORMULATION}

A uniform flow of air with velocity $U_{\infty}^{*}$ and temperature $T_{\infty}^{*}$ past an infinitely thin flat plate is considered. The Mach number is defined as $M \equiv U_{\infty}^{*} / c_{\infty}^{*}=\mathcal{O}(1)$, where $c_{\infty}^{*}=\sqrt{\gamma \mathcal{R} T_{\infty}^{*}}$ is the 


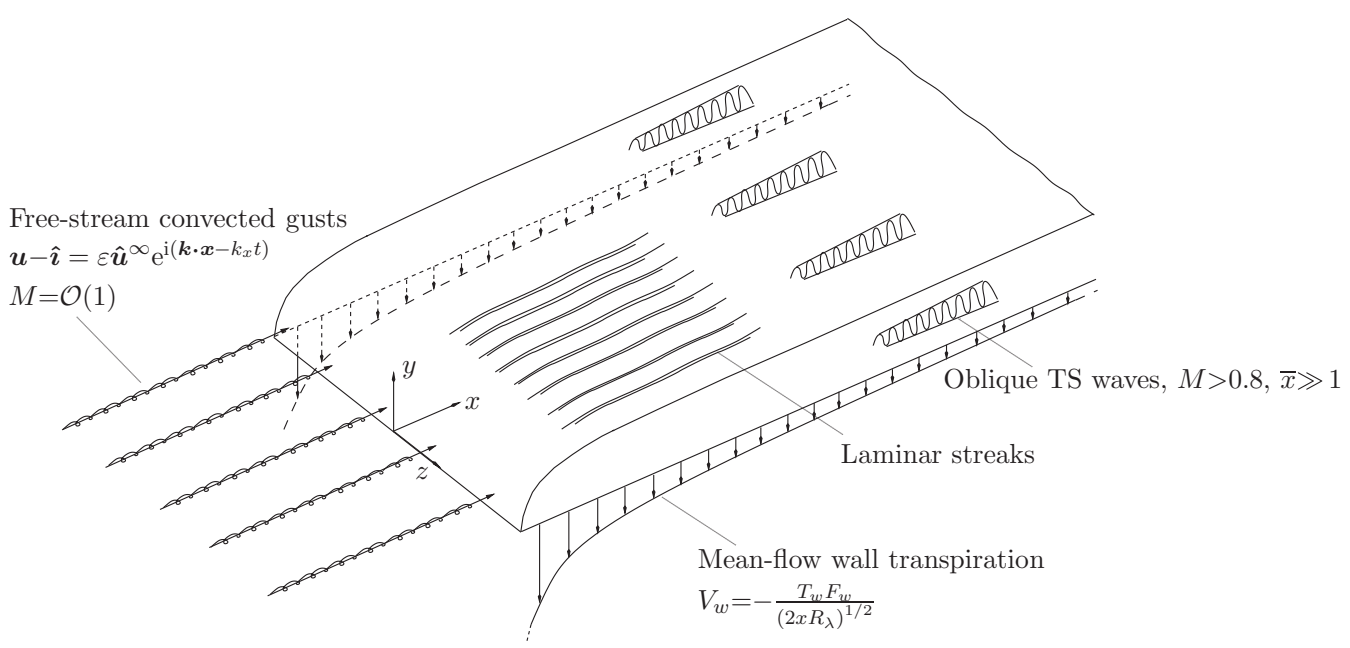

FIG. 1. Schematic of the physical domain.

speed of sound in the free stream, $\gamma=1.4$ is the ratio of the specific heats and $\mathcal{R}=287.05$ $\mathrm{Nm} \mathrm{kg}{ }^{-1} \mathrm{~K}^{-1}$ is the specific gas constant for air. Mean-flow wall suction is applied and the wall is assumed to be adiabatic. Both subsonic $(M<1)$ and supersonic $(M>1)$ conditions are investigated.

The flow is described in a Cartesian coordinate system, where $x^{*}, y^{*}$, and $z^{*}$ define the streamwise, wall-normal, and spanwise directions, respectively, and the leading edge of the plate is located at $x^{*}=y^{*}=0$. The symbol $*$ indicates a dimensional quantity and quantities not marked by any symbol are dimensionless. A schematic of the physical domain is displayed in Figure 1. In the free stream, the spatial coordinates $x^{*}, y^{*}, z^{*}$ are scaled by $\lambda_{z}^{*}$, the spanwise wavelength of the gust, and time is scaled by $\lambda_{z}^{*} / U_{\infty}^{*}$. The velocities and the temperature are scaled by $U_{\infty}^{*}$ and $T_{\infty}^{*}$. The pressure is normalized by $\rho_{\infty}^{*} U_{\infty}^{* 2}$ (where $\rho_{\infty}^{*}$ is the constant free-stream density). The density $\rho^{*}$ and the dynamic viscosity $\mu^{*}$ are scaled by their respective free-stream values.

Superimposed on $U_{\infty}^{*}$ are small, homogeneous, statistically stationary vortical fluctuations of the convected gust type, i.e., perturbations which are advected at a velocity $U_{\infty}^{*}$. As these individual modes are assumed of small amplitude and do not interact with one another, it is sufficient to consider a single disturbance of the form

$$
\boldsymbol{u}-\hat{\boldsymbol{\imath}}=\varepsilon \hat{\boldsymbol{u}}^{\infty} \mathrm{e}^{\mathrm{i}\left(k_{x} x+k_{y} y+k_{z} z-k_{x} t\right)}+c . c .,
$$

where $\boldsymbol{u}$ is the free-stream velocity vector, $\hat{\boldsymbol{\imath}}$ is the unit vector along the streamwise direction representing the normalized mean free-stream velocity, $\varepsilon \ll 1$ indicates the gust amplitude, $\hat{\boldsymbol{u}}^{\infty}=\left\{\hat{u}^{\infty}, \hat{v}^{\infty}, \hat{w}^{\infty}\right\}=\mathcal{O}(1)$, and c.c. denotes the complex conjugate. The wavenumbers are scaled by the inverse of the spanwise wavelength $\lambda_{z}^{*}$. Our primary focus will be on low-frequency disturbances with $k_{x} \ll k_{y}, k_{z}$ because these disturbances penetrate the most into the boundary layer to form the laminar streaks. A Reynolds number $R_{\lambda} \equiv U_{\infty}^{*} \lambda_{z}^{*} / \nu_{\infty}^{*} \gg 1$ is defined, where $v_{\infty}^{*}$ is the kinematic viscosity of the fluid in the free stream. It follows from the continuity equation that

$$
k_{x} \hat{u}^{\infty}+k_{y} \hat{v}^{\infty}+k_{z} \hat{w}^{\infty}=0 .
$$

Downstream locations at which $\delta_{99}^{*}=\mathcal{O}\left(\lambda_{z}^{*}\right)$ are investigated, where $\delta_{99}^{*}$ is the boundary layer thickness, defined as the wall-normal location where the value of the streamwise velocity attains $99 \%$ of the mean free-stream velocity value. At these locations $x / R_{\lambda}=\mathcal{O}(1)$, which means that the spanwise diffusion is of the same order as that in the wall-normal direction. A distinguished scaling $k_{x}=\mathcal{O}\left(R_{\lambda}^{-1}\right)$ is investigated, as the laminar streaks evolve downstream on a length scale which is comparable with the gust streamwise wavelength. Due to the disparity between the spanwise and streamwise scales, $\mathcal{O}(\varepsilon)$ free-stream fluctuations generate $\mathcal{O}\left(\varepsilon / k_{x}\right)$ streamwise velocity disturbances within the boundary layer. As the disturbance amplitude is assumed to be much smaller than the mean flow amplitude, the equations governing the disturbances can be linearized. The condition for 
linearization is $\varepsilon / k_{x} \ll 1$ or equivalently $\varepsilon R_{\lambda} \ll 1$. The reader should refer to LWG for a thorough derivation of these scaling relationships.

\section{A. The boundary-layer mean flow}

The steady compressible equations for conservation of mass, momentum, and energy within the boundary layer are cast into a more compact form by means of the Dorodnitsyn-Howarth coordinate transformation (see Stewartson ${ }^{36}$ for details)

$$
\bar{Y}=\bar{Y}(x, y) \equiv \int_{0}^{y} \rho(x, \breve{y}) \mathrm{d} \breve{y} .
$$

In the absence of a mean-flow pressure gradient, a similarity solution exists in terms of the variable

$$
\eta \equiv \bar{Y}\left(\frac{R_{\lambda}}{2 x}\right)^{1 / 2}=\bar{Y} \lambda_{z}^{*} \sqrt{\frac{U_{\infty}^{*}}{2 v_{\infty}^{*} x^{*}}} .
$$

In terms of $\eta$, the mean flow solution can be expressed as

$$
U=F^{\prime}(\eta), V=\left(2 x R_{\lambda}\right)^{-1 / 2}\left(\eta_{c} T F^{\prime}-T F\right), T=T(\eta),
$$

where $U$ and $V$ are the mean streamwise and wall-normal velocities, and $T$ is the mean temperature. The prime denotes differentiation with respect to $\eta$, and

$$
\eta_{c} \equiv \frac{1}{T} \int_{0}^{\eta} T(\breve{\eta}) \mathrm{d} \breve{\eta}
$$

The $x$-momentum and energy equations can be written as a coupled system of nonlinear ordinary differential equations for $F$ and $T^{36}$

$$
\begin{array}{r}
{\left[(\mu / T) F^{\prime \prime}\right]^{\prime}+F F^{\prime \prime}=0,} \\
\operatorname{Pr}^{-1}\left[(\mu / T) T^{\prime}\right]^{\prime}+F T^{\prime}+(\gamma-1) M^{2}(\mu / T) F^{\prime \prime 2}=0,
\end{array}
$$

where the Prandtl number for air is $\operatorname{Pr}=0.7$. The dependence of the viscosity upon temperature satisfies the power law

$$
\mu=T^{\omega},
$$

where $\omega=0.76$. This relation is preferred to the simpler Chapman law for which $\omega=1$, as it captures the dependence of viscosity upon temperature more accurately in the Mach number range of interest $(M \leq 4) \cdot{ }^{34}$ In the far field

$$
F^{\prime} \rightarrow 1, T \rightarrow 1, \quad \text { as } \eta \rightarrow \infty,
$$

so that the boundary layer velocity and temperature profiles approach their free-stream values far away from the flat plate. The displacement constant $\beta_{c}$ is

$$
\beta_{c}=\lim _{\eta \rightarrow \infty}(\eta-F),
$$

and it depends on both $M$ and the wall suction. On the flat plate

$$
F(0)=F_{w}, F^{\prime}(0)=0, \quad \text { and } T^{\prime}(0)=0,
$$

where the first boundary condition synthesizes the wall transpiration, the second one the streamwise no-slip condition, and the third one the adiabatic wall condition. The wall velocity $V_{w}$ and wall temperature $T_{w}$ are related through

$$
V_{w}=-\frac{T_{w} F_{w}}{\left(2 x R_{\lambda}\right)^{1 / 2}}=-T_{w} F_{w}\left(\frac{v_{\infty}^{*}}{U_{\infty}^{*} 2 x \lambda_{z}^{*}}\right)^{1 / 2},
$$


with $F_{w}>0$ indicating wall suction and $F_{w}<0$ indicating wall blowing. Such a distribution of wall transpiration guarantees a similarity form of the equations where $F_{w}$ alone defines the transpiration intensity, and consequently the wall suction effect can be evaluated through changes in $F_{w}$. The mean boundary layer equations (4) are solved numerically by the method described in Cebeci. ${ }^{7}$

\section{Limit of large mean-flow suction}

It is worth investigating whether there are limits in which the mean flow can be more conveniently represented. For large wall suction $\left(F_{w} \gg 1\right)$, the boundary layer becomes very thin $(\eta \ll 1)$. When $F_{w} \eta=\mathcal{O}(1)$, a new wall-normal coordinate,

$$
N=F_{w} \eta=-\frac{\bar{Y} \lambda_{z}^{*} V_{w}^{*} T_{\infty}^{*}}{\nu_{\infty}^{*} T_{w}^{*}}
$$

is defined. In terms of this new coordinate, the mean flow equations (4) become

$$
\begin{aligned}
& \frac{\mathrm{d}}{\mathrm{d} N}\left(\frac{\mu}{T} \frac{\mathrm{d} U}{\mathrm{~d} N}\right)+\frac{\mathrm{d} U}{\mathrm{~d} N}=0 \\
& \frac{1}{\operatorname{Pr}} \frac{\mathrm{d}}{\mathrm{d} N}\left(\frac{\mu}{T} \frac{\mathrm{d} T}{\mathrm{~d} N}\right)+\frac{\mathrm{d} T}{\mathrm{~d} N}+\frac{(\gamma-1) M^{2} \mu}{T}\left(\frac{\mathrm{d} U}{\mathrm{~d} N}\right)^{2}=0
\end{aligned}
$$

while the wall-normal velocity and temperature are related by $V / T=V_{w} / T_{w}$.

Equation (8a) is integrated with respect to $N$ and the boundary condition (6) is used to obtain an expression for the large suction asymptotic velocity gradient $F_{\text {asy }}^{\prime \prime}$, as follows:

$$
\frac{\mathrm{d} U}{\mathrm{~d} N}=\frac{F_{\text {asy }}^{\prime \prime}}{F_{w}}=\frac{T}{\mu} \exp \left(-\int_{0}^{N} \frac{T}{\mu} \mathrm{d} \tilde{N}\right) .
$$

Further integration and substitution for the definition of $V_{w}$ from (7) give expressions for the streamwise and wall-normal velocities with the form

$$
\begin{gathered}
U=F_{\text {asy }}^{\prime}=1-\exp \left(-\int_{0}^{N} \frac{T}{\mu} \mathrm{d} \tilde{N}\right), \\
V=-\frac{T F_{w}}{\left(2 x R_{\lambda}\right)^{1 / 2}},
\end{gathered}
$$

while the temperature is governed by

$$
\frac{\mu}{\operatorname{Pr} T} \frac{\mathrm{d} T}{\mathrm{~d} N}+T=1+\frac{\gamma-1}{2} M^{2}(1-U)^{2} .
$$

Analytical solutions for the wall-shear stress and the wall temperature are found from (9) and (10), respectively, as follows:

$$
\begin{aligned}
\left.\frac{\mathrm{d} U}{\mathrm{~d} N}\right|_{N=0} & =\frac{F_{\text {asy }}^{\prime \prime}(0)}{F_{w}}, \\
T_{w} & =1+\frac{M^{2}}{2}(\gamma-1) .
\end{aligned}
$$

The asymptotic limit of the displacement constant $\beta_{c}$ is

$$
\left.\lim _{\eta \rightarrow \infty} \beta_{c}\right|_{F_{w} \gg 1}=-F_{w}+\frac{\mu_{w}}{T_{w} F_{w}}=-F_{w}+\frac{1}{F_{\text {asy }}^{\prime \prime}(0)} .
$$

As verified by Ricco and Dilib ${ }^{30}$ in the incompressible case, the asymptotic values of $\beta_{c}$ and $F_{\text {asy }}^{\prime \prime}(0)$ are in excellent agreement with the numerically computed values when $F_{w} \gg 1$. For $F_{w}=3$, the 


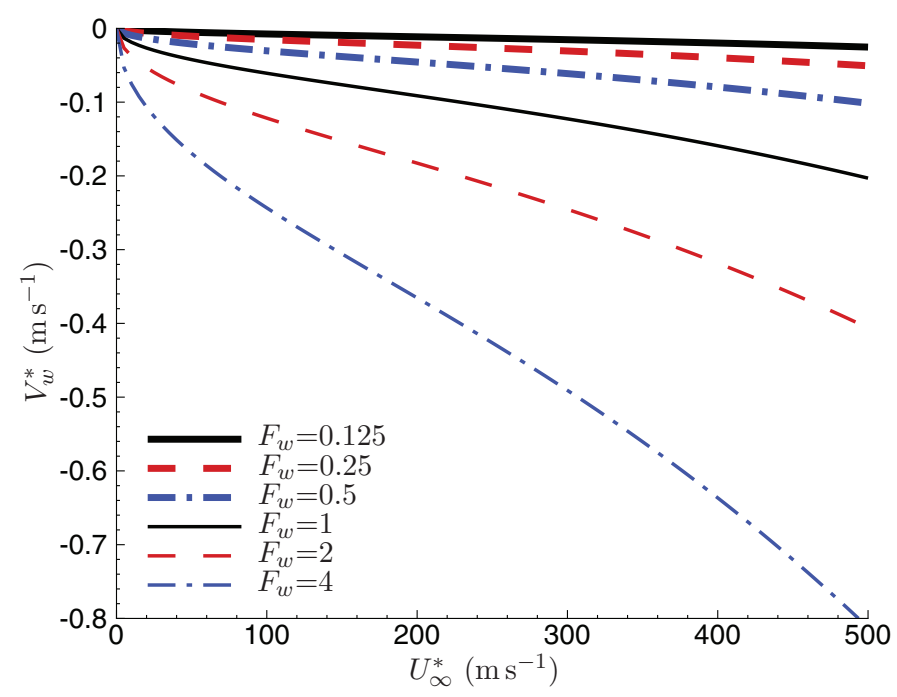

FIG. 2. Wall suction velocities $V_{w}^{*}$ as a function of the free-stream velocity $U_{\infty}^{*}$ for different $F_{w}$ for aircraft cruise conditions along a wing at $x^{*}=0.5 \mathrm{~m}$ with $p_{\infty}^{*}=30000 \mathrm{~Pa}$ and $T_{\infty}^{*}=238.15 \mathrm{~K}$. At these conditions $M=1$ corresponds to $U_{\infty}^{*}=311 \mathrm{~m} \mathrm{~s}^{-1}$.

relative errors between the large suction wall temperature (11b) and its numerically computed counterpart are $0.29 \%$ at $M=0.8$ and $1.59 \%$ at $M=3$. Even without suction the relative error between the large suction wall temperature and the numerically computed value at $M=0.8$ is just $1.86 \%$. The good agreement even in the no-suction case is due to (11b) being also valid in the no-suction case when $\operatorname{Pr}=1 .{ }^{36} \mathrm{An}$ expression for $V_{w}$ can be obtained from (7) and (11b),

$$
V_{w}=-F_{w}\left(\frac{v_{\infty}^{*}}{U_{\infty}^{*} 2 x \lambda_{z}^{*}}\right)^{1 / 2}\left[1+\frac{M^{2}}{2}(\gamma-1)\right],
$$

which can be used to compute the dimensional wall suction velocity $V_{w}^{*}$ in cruising flight conditions. Figure 2 shows that the values of wall suction velocity are much smaller than the cruising speed. It is shown in Sec. III that, for an aircraft cruising at $M=0.85$, levels of wall suction corresponding to about $0.05 \%\left(F_{w} \approx 1\right)$ of the free-stream velocity produce significant reductions of the disturbance energy within the boundary layer, which renders wall suction an attractive method for technological implementation at high speeds.

Equation (10) cannot be solved analytically when the power law model for viscosity, given by (5), is used. However, when the Chapman viscosity law, $\mu=T$, is applied, the solutions for $U$ and $T$ are

$$
\begin{aligned}
& U=1-\mathrm{e}^{-N}, \\
& T=1+\frac{(\gamma-1) M^{2}}{2}\left(\frac{\operatorname{Pr} \mathrm{e}^{-2 N}-2 \mathrm{e}^{-\operatorname{Pr} N}}{\operatorname{Pr}-2}\right),
\end{aligned}
$$

which is analogous to the asymptotic solution (46) in Lew and Fanucci ${ }^{23}$ for uniform suction. The asymptotic Chapman law solution is a simple model, while the numerically computed power law solution is more realistic. It is therefore useful to compare the results obtained through these models and investigate under which conditions the asymptotic approach produces a valid approximation. Graphs (a) and (b) in Figure 3 show the profiles of mean velocity and temperature for $M=0.8$, computed numerically by using the power law for viscosity for different $F_{w}$. Both the velocity and temperature profiles are brought closer to the wall, i.e., the kinematic and thermal boundary layers become thinner as the transpiration velocity increases. The wall temperature increases with 

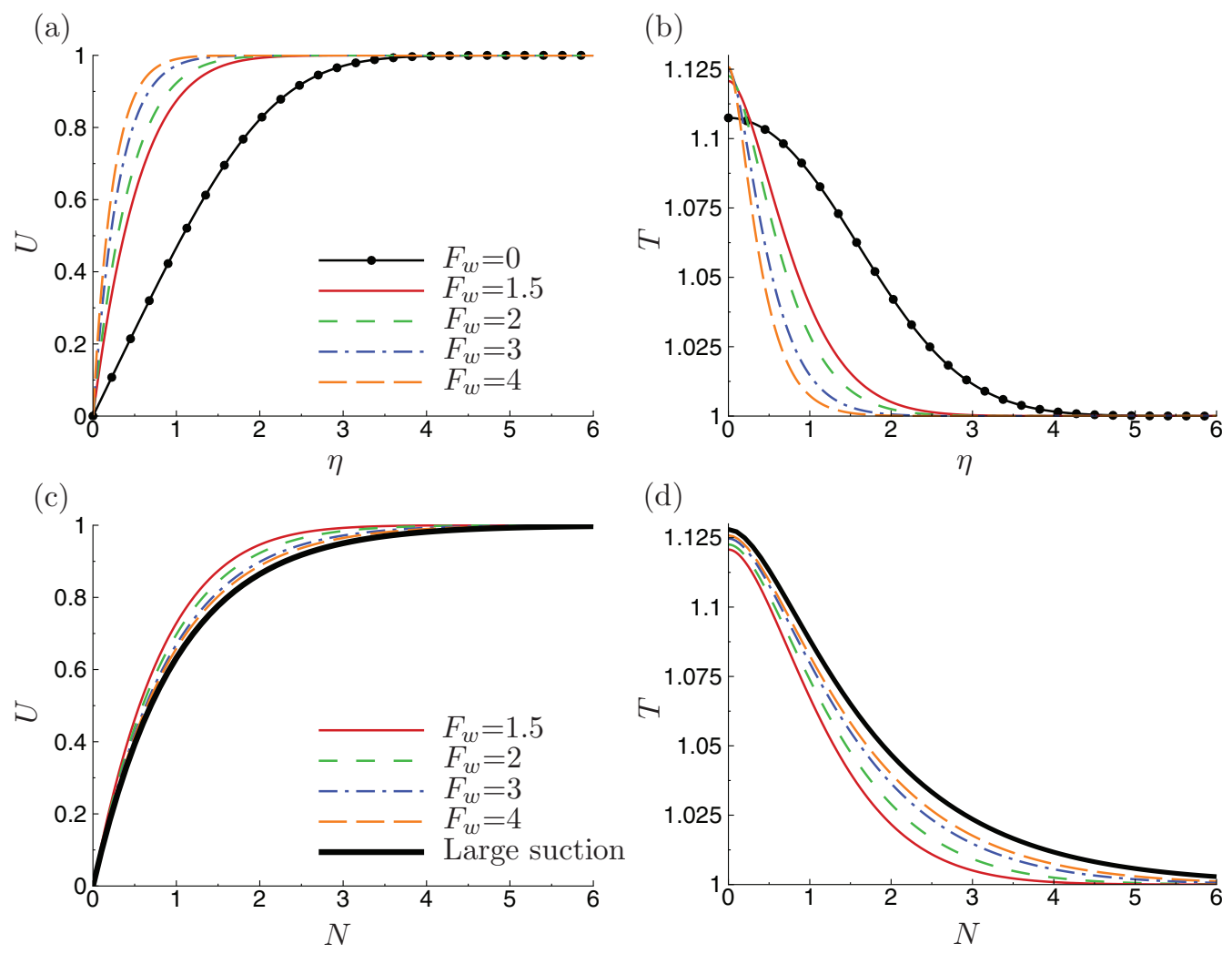

FIG. 3. Profiles of (a) the streamwise mean flow $U$ and (b) the temperature $T$ at $M=0.8$ calculated through both the large wall suction asymptotic solutions with the Chapman viscosity law for different $F_{w}$ (expressions (12) - thick solid line) and the full numerical solution of (4) with a power-law viscosity.

the suction intensity. The same profiles are replotted in graphs (c) and (d) as functions of $N$ and compared with the asymptotic Chapman law analytical solutions (12). The agreement between the asymptotic and numerical profiles improves as the wall suction increases and satisfactory agreement is obtained even with quite moderate suction. In addition to the errors related to the assumption of large wall suction, differences between the numerical and asymptotic profiles also result from the different viscosity models.

Table I quantifies the maximum percentage relative error between the numerical and asymptotic profiles,

$$
E_{\max }(\%) \equiv \max _{\eta}\left[100 \cdot\left|\frac{f_{n}(\eta)-f_{a}(\eta)}{f_{n}(\eta)}\right|\right],
$$

where $f$ represents $U$ or $T$ and the subscripts $n$ and $a$ indicate the numerical and asymptotic solutions, respectively. At $M=0.8$, the errors indicate that the asymptotic profiles are a good proxy for the numerical profiles with either viscosity laws, with the temperature profiles showing an excellent

TABLE I. Maximum percentage relative errors $E_{\max }$, defined in (13), between the asymptotic large suction profiles and the full numerically computed profiles for $F_{w}=4$.

\begin{tabular}{llcc}
\hline \hline Quantity & $M$ & $E_{\max }$ Chapman & $E_{\max }$ power law \\
\hline$U$ & 0.8 & $2.8 \%$ & $5.4 \%$ \\
$T$ & 0.8 & $0.6 \%$ & $0.7 \%$ \\
$U$ & 3 & $2.8 \%$ & $23 \%$ \\
$T$ & 3 & $6.4 \%$ & $15.7 \%$ \\
\hline \hline
\end{tabular}


agreement. The asymptotic solution is a convenient substitute for the Chapman law numerical solution at $M=3$, but is less useful as a replacement for the power law numerical solution, indicating that in this case the primary cause of error is the change in the viscosity model rather than the asymptotic approximation. The larger discrepancies in the temperature profiles occurring at larger Mach number are due to a stronger dependence of temperature on $U$ through viscous aerodynamic heating as $M$ increases.

\section{B. The boundary-layer disturbance flow}

The boundary layer flow is decomposed as the sum of the mean flow, studied in Sec. II A, and the small-amplitude perturbation flow, as follows:

$$
\begin{gathered}
\{u, v, w, \tau, p\}=\{U, V, 0, T,-1 / 2\} \\
+\varepsilon\left\{\bar{u}_{0}(\bar{x}, \eta), k_{x}^{*}\left(\frac{2 v_{\infty}^{*} x^{*}}{U_{\infty}^{*}}\right)^{1 / 2} \bar{v}_{0}(\bar{x}, \eta), \bar{w}_{0}(\bar{x}, \eta), \bar{\tau}_{0}(\bar{x}, \eta), \bar{p}_{0}(\bar{x}, \eta)\right\} \mathrm{e}^{\mathrm{i}\left(k_{z} z-k_{x} t\right)}+c . c .,
\end{gathered}
$$

where $\left\{\bar{u}_{0}, \bar{v}_{0}, \bar{w}_{0}, \bar{\tau}_{0}, \bar{p}_{0}\right\}$ are the disturbance velocities, temperature, and pressure. The streamwise coordinate is scaled by the gust streamwise wavenumber $k_{x}^{*}=2 \pi / \lambda_{x}^{*}$, i.e., $\bar{x}=k_{x} x=2 \pi x^{*} / \lambda_{x}^{*}$ $=\mathcal{O}(1)$, where $\lambda_{x}^{*}$ is the gust streamwise wavelength.

Following Gulyaev et al. ${ }^{15}$ and LWG, the solution is expanded as a weighted sum of $\left\{\bar{u}^{(0)}, \bar{v}^{(0)}, \bar{w}^{(0)}, \bar{\tau}^{(0)}, \bar{p}^{(0)}\right\}$ and $\{\bar{u}, \bar{v}, \bar{w}, \bar{\tau}, \bar{p}\}$. The evolution of the former components in the incompressible case has been considered by $\operatorname{Ricco}^{29}$ and it is dominant in the outer part of the boundary layer. In the present work, the focus is on the latter components as they dominate the core of the boundary layer and exhibit the streak growth downstream. Their evolution is governed by the compressible linearized unsteady boundary-region (CLUBR) equations, which read ${ }^{33}$

Continuity equation

$$
\begin{gathered}
\frac{\partial \bar{u}}{\partial \bar{x}}+\frac{\eta_{c}}{2 \bar{x}}\left(\frac{T^{\prime}}{T} \bar{u}-\frac{\partial \bar{u}}{\partial \eta}\right)-\frac{T^{\prime}}{T^{2}} \bar{v}+\frac{1}{T} \frac{\partial \bar{v}}{\partial \eta}+\bar{w}+\left(\frac{\mathrm{i}}{T}-\frac{F T^{\prime}}{2 \bar{x} T^{2}}\right) \bar{\tau}-\frac{F^{\prime}}{T} \frac{\partial \bar{\tau}}{\partial \bar{x}}+\frac{F}{2 \bar{x} T} \frac{\partial \bar{\tau}}{\partial \eta}=0, \\
x \text {-Momentum equation } \\
\left(-\mathrm{i}-\frac{\eta_{c} F^{\prime \prime}}{2 \bar{x}}+\mu \kappa^{2} T\right) \bar{u}+F^{\prime} \frac{\partial \bar{u}}{\partial \bar{x}}-\frac{1}{2 \bar{x}}\left(F+\frac{\mu^{\prime} T^{\prime}}{T}-\frac{\mu T^{\prime}}{T^{2}}\right) \frac{\partial \bar{u}}{\partial \eta}-\frac{\mu}{2 \bar{x} T} \frac{\partial^{2} \bar{u}}{\partial \eta^{2}}+\frac{F^{\prime \prime}}{T} \bar{v} \\
+\left(\frac{F F^{\prime \prime}-\mu^{\prime \prime} T^{\prime} F^{\prime \prime}-\mu^{\prime} F^{\prime \prime \prime}}{2 \bar{x} T}+\frac{\mu^{\prime} F^{\prime \prime} T^{\prime}}{2 \bar{x} T^{2}}\right) \bar{\tau}-\frac{\mu^{\prime} F^{\prime \prime}}{2 \bar{x} T} \frac{\partial \bar{\tau}}{\partial \eta}=0,
\end{gathered}
$$

y-Momentum equation

$$
\begin{gathered}
\frac{1}{4 \bar{x}^{2}}\left[F T+\eta_{c}\left(F T^{\prime}-T F^{\prime}\right)-\eta_{c}^{2} F^{\prime \prime} T\right] \bar{u}+\frac{\mu^{\prime} T^{\prime}}{3 \bar{x}} \frac{\partial \bar{u}}{\partial \bar{x}}-\frac{\mu}{6 \bar{x}} \frac{\partial^{2} \bar{u}}{\partial \eta \partial \bar{x}}+\frac{1}{12 \bar{x}^{2}}\left[\mu+\eta_{c} T\left(\frac{\mu}{T}\right)^{\prime}\right] \frac{\partial \bar{u}}{\partial \eta} \\
+\frac{\eta_{c} \mu}{12 \bar{x}^{2}} \frac{\partial^{2} \bar{u}}{\partial \eta^{2}}+\left(-\mathrm{i}+\mu \kappa^{2} T+\frac{F^{\prime}}{2 \bar{x}}+\frac{\eta_{c} F^{\prime \prime}}{2 \bar{x}}-\frac{F T^{\prime}}{2 \bar{x} T}\right) \bar{v}+F^{\prime} \frac{\partial \bar{v}}{\partial \bar{x}} \\
-\frac{1}{\bar{x}}\left(\frac{F}{2}+\frac{2 \mu^{\prime} T^{\prime}}{3 T}-\frac{2 \mu T^{\prime}}{3 T^{2}}\right) \frac{\partial \bar{v}}{\partial \eta}-\frac{2 \mu}{3 \bar{x} T} \frac{\partial^{2} \bar{v}}{\partial \eta^{2}}+\frac{\mu^{\prime} T^{\prime}}{3 \bar{x}} \bar{w}-\frac{\mu}{6 \bar{x}} \frac{\partial \bar{w}}{\partial \eta}+\frac{1}{2 \bar{x}} \frac{\partial \bar{p}}{\partial \eta} \\
+\frac{1}{4 \bar{x}^{2}}\left\{\eta_{c}\left[\left(F F^{\prime}\right)^{\prime}-T\left(\frac{\mu^{\prime} F^{\prime \prime}}{T}\right)^{\prime}\right]-F F^{\prime}-\frac{F^{2} T^{\prime}}{T}-\mu^{\prime} F^{\prime \prime}+\frac{4}{3}\left(\frac{\mu^{\prime} T^{\prime} F}{T}\right)^{\prime}\right\} \bar{\tau} \\
-\frac{\mu^{\prime} F^{\prime \prime}}{2 \bar{x}} \frac{\partial \bar{\tau}}{\partial \bar{x}}-\left(\frac{\eta_{c} \mu^{\prime} F^{\prime \prime}}{4 \bar{x}^{2}}-\frac{\mu^{\prime} T^{\prime} F}{3 \bar{x}^{2} T}\right) \frac{\partial \bar{\tau}}{\partial \eta}=0
\end{gathered}
$$


z-Momentum equation

$$
\begin{aligned}
& -\frac{\eta_{c} \mu^{\prime} T^{\prime} T \kappa^{2}}{2 \bar{x}} \bar{u}+\frac{\mu T \kappa^{2}}{3} \frac{\partial \bar{u}}{\partial \bar{x}}-\frac{\eta_{c} \mu T \kappa^{2}}{6 \bar{x}} \frac{\partial \bar{u}}{\partial \eta}+\mu^{\prime} T^{\prime} \kappa^{2} \bar{v}+\frac{\mu \kappa^{2}}{3} \frac{\partial \bar{v}}{\partial \eta}-\left(\mathrm{i}-\frac{4 \mu T \kappa^{2}}{3}\right) \bar{w} \\
& +F^{\prime} \frac{\partial \bar{w}}{\partial \bar{x}}-\frac{1}{2 \bar{x}}\left(F+\frac{\mu^{\prime} T^{\prime}}{T}-\frac{\mu T^{\prime}}{T^{2}}\right) \frac{\partial \bar{w}}{\partial \eta}-\frac{\mu}{2 \bar{x} T} \frac{\partial^{2} \bar{w}}{\partial \eta^{2}}+\frac{\mu^{\prime} F T^{\prime} \kappa^{2}}{3 \bar{x}} \bar{\tau}-\kappa^{2} T \bar{p}=0,
\end{aligned}
$$

Energy equation

$$
\begin{aligned}
-\frac{\eta_{c} T^{\prime}}{2 \bar{x}} \bar{u} & -\frac{M^{2}(\gamma-1) \mu F^{\prime \prime}}{\bar{x} T} \frac{\partial \bar{u}}{\partial \eta}+\frac{T^{\prime}}{T} \bar{v}+\left[-\mathrm{i}+\frac{F T^{\prime}}{2 \bar{x} T}-\frac{1}{2 \operatorname{Pr} \bar{x}}\left(\frac{\mu^{\prime} T^{\prime}}{T}\right)^{\prime}-\frac{M^{2}(\gamma-1) \mu^{\prime} F^{\prime \prime 2}}{2 \bar{x} T}\right. \\
& \left.+\frac{\mu \kappa^{2} T}{\operatorname{Pr}}\right] \bar{\tau}+F^{\prime} \frac{\partial \bar{\tau}}{\partial \bar{x}}-\frac{1}{2 \bar{x}}\left(F+\frac{2 \mu^{\prime} T^{\prime}}{\operatorname{Pr} T}-\frac{\mu T^{\prime}}{\operatorname{Pr} T^{2}}\right) \frac{\partial \bar{\tau}}{\partial \eta}-\frac{\mu}{2 \operatorname{Pr} \bar{x} T} \frac{\partial^{2} \bar{\tau}}{\partial \eta^{2}}=0
\end{aligned}
$$

where

$$
\kappa_{y} \equiv \sqrt{\frac{2 \pi \nu_{\infty}^{*} \lambda_{x}^{*}}{U_{\infty}^{*}}} \frac{1}{\lambda_{y}^{*}}=\mathcal{O}(1), \quad \text { and } \quad \kappa \equiv \sqrt{\frac{2 \pi \nu_{\infty}^{*} \lambda_{x}^{*}}{U_{\infty}^{*}}} \frac{1}{\lambda_{z}^{*}}=\mathcal{O}(1),
$$

are the scaled wall-normal and spanwise wavenumbers, respectively. At the wall, the no-slip wall boundary conditions are applied to the streamwise and spanwise disturbance velocities, while the no-penetration conditions are applied to the wall-normal disturbance velocity. Through the use of Darcy's law, Gustavsson ${ }^{16}$ has shown that the wall-normal disturbance velocity does indeed satisfy the no-penetration condition, provided the surface permeability is low, a situation often encountered in experiments. ${ }^{12}$ The adiabatic wall condition is used for the temperature disturbance. As the free stream is approached, the boundary-layer fluctuations are matched with the convective gust disturbances, so that the outer boundary conditions are

$$
\begin{aligned}
\bar{u} & \rightarrow 0, \\
\bar{\tau} & \rightarrow 0, \\
{\left[\frac{\partial}{\partial \eta}+|\kappa|(2 \bar{x})^{1 / 2}\right]\{\bar{v}, \bar{w}, \bar{p}\} } & \rightarrow\left\{-1, \mathrm{i} \kappa_{y}(2 \bar{x})^{1 / 2}, 0\right\} \mathrm{e}^{\mathrm{i}\left(\bar{x}+\kappa_{y}(2 \bar{x})^{1 / 2} \bar{\eta}\right)} \mathrm{e}^{-\left(\kappa_{y}^{2}+\kappa^{2}\right) \bar{x}},
\end{aligned}
$$

as $\eta \rightarrow \infty$, where $\bar{\eta} \equiv \eta-\beta_{c}$. Further details on the derivation of the outer boundary conditions can be found in LWG and RW. Note that the scaled wall-normal wavenumber $\kappa_{y}$ only appears in the outer boundary conditions and not in the boundary-region equations. This is because the wall-normal length scale of the outer flow disturbance is $\lambda_{y}^{*}$, while within the boundary layer the characteristic length scale of the fluctuations is the boundary-layer thickness itself, so $\lambda_{y}^{*}$ becomes irrelevant there.

The boundary-region equations (4.1)-(4.5) of RW are parabolic in the $\bar{x}$ direction and elliptic in the $z$ direction. Given the initial conditions (4.12)-(4.16) of RW, solutions can be obtained by marching downstream and applying the wall boundary condition and the conditions (19) at the outer edge. A second-order finite-difference scheme, which is central in $\eta$ and backward implicit in $\bar{x}$, is used. The pressure disturbance is computed on a grid staggered in the $\eta$ direction with respect to the grid for the velocity components to eliminate the pressure decoupling phenomenon, which occurs if the two grids coincide. The resulting linear system is solved by a standard block-elimination algorithm. $^{7}$

\section{RESULTS}

The effects of wall suction on the velocity and temperature fluctuations are now investigated. It is assumed that variations of the Mach number $M$ are due only to the changes in $U_{\infty}^{*}$, while $T_{\infty}^{*}$ remains constant. It is also assumed that the modulus of the gust vector remains equal to unity, i.e., 
$\left[\left(\hat{u}^{\infty}\right)^{2}+\left(\hat{v}^{\infty}\right)^{2}+\left(\hat{w}^{\infty}\right)^{2}\right]^{1 / 2}=1$, and that, when $M$ varies, $k_{x}^{*}$ is kept constant, $\hat{u}^{\infty}=1$, and $k_{z}=$ $k_{y}$, so that the properties of the gust are fully specified by use of (2). In typical flight conditions, for which $U_{\infty}^{*}=225 \mathrm{~m} / \mathrm{s}$ and $v_{\infty}^{*}=35 \times 10^{-6} \mathrm{~m}^{2} / \mathrm{s}$, and for a spanwise length scale of about $\lambda_{z}^{*}=1 \mathrm{~mm}$, a spanwise wavenumber $\kappa=1$ gives a streamwise wavelength of about $1 \mathrm{~m}$ and $k_{x}^{*}=6.14 \mathrm{~m}^{-1}$. The mean flow profiles for large wall suction calculated in Sec. II A could be used here. However, the most interesting behaviour is obtained with comparatively small suction levels and therefore the mean flow has been calculated numerically using (4) and (5).

\section{A. Klebanoff modes for $\kappa \gg 1$}

LWG found that the incompressible linearized unsteady boundary-region equations possess an asymptotic solution for large $\kappa$, for which both the streak amplitude and streamwise development are rescaled by $\kappa^{2}$. Ricco, Tran, and $\mathrm{Ye}^{32}$ have numerically verified that the asymptotic solution also holds for streamwise velocity component with wall heating and cooling for $M=0$, with the collapse being more effective in the wall heating case when the amplitude of the disturbance is smaller. The scaling for the velocity components is given by equations (5.32)-(5.34) in LWG and the asymptotic solution for the temperature disturbance has the form $\bar{\tau}=\kappa^{-2} \hat{\tau}\left(\kappa^{2} \bar{x}, \eta ; \kappa_{y} / \kappa\right)$. The disturbances satisfy the compressible linearized steady boundary-region equations when $\kappa \gg 1$ and decay rapidly after the initial algebraic growth because of the intense viscous effects produced by the large spanwise wavenumber. The asymptotically scaled velocity and temperature disturbance profiles are shown in Figure 4, without (left) and with (right) wall suction. Both the streamwise velocity and temperature profiles tend to collapse onto one another as $\kappa$ grows. Good agreement is obtained even for values of $\kappa$ as low as $\kappa=0.5$, with only a $10 \%$ deviation for $\kappa=0.25$. The profiles collapse better onto the
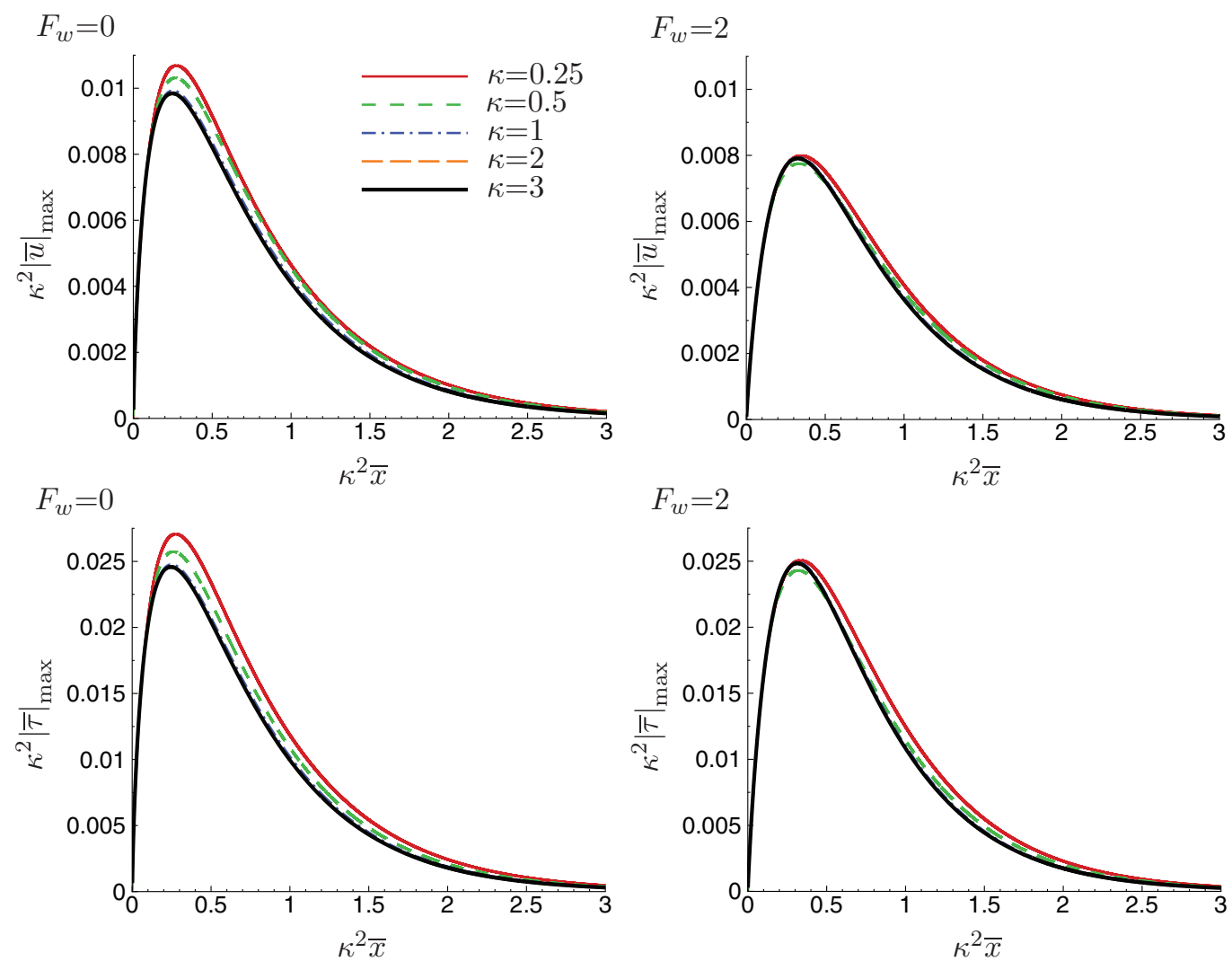

FIG. 4. Large $\kappa$ asymptotic scaled solutions for $M=3$ for the peak streamwise disturbance velocity (top row) and the peak disturbance temperature (bottom row). 
asymptotic profile in the wall suction case as the steady boundary-region equations more accurately represent the disturbance dynamics when the boundary layer is thinner. The attenuating effect of wall suction is moderate on the streamwise velocity. The temperature profiles are only slightly attenuated with wall suction, with the effect becoming smaller as $\kappa$ increases and negligible in the limit $\kappa \gg 1$. In subsonic cases and in the incompressible limit, the amplitude of the disturbances is also attenuated by wall suction and the collapse also occurs for $\kappa$ values as small as 0.25 .

\section{B. Klebanoff modes for $\kappa=\mathcal{O}(1)$}

The effect of wall transpiration on the streaks with $\kappa=1$ at $M=3$ is shown in Figures 5 (suction) and 6 (blowing), respectively. At this Mach number, the thermal streaks dominate as the amplitude is approximately twice that of the kinematic streaks, and the low wall suction levels actually increase the streak amplitude. Kinematic streaks reach their peak for $F_{w} \approx 0.25$ and thermal streaks for $F_{w} \approx$ 1. Figure 5 reports that there is an initial reduction in maximum streak amplitude with blowing for about $-0.6<F_{w}<0$. For $F_{w}<-0.6$, the maximum streak increases sharply above the no-suction case from locations which are very close to the leading edge. The location of the maximum for both velocity and thermal disturbances increases as the blowing intensity increases.

Figure 7 instead shows that, in the subsonic regime, kinematic streaks are dominant as their magnitude is about one order of magnitude larger than that of the thermal streaks. For $M=0.8$, increasing wall suction monotonically reduces the streak growth rate and amplitude, with the attenuating effect being twice as intense as for $M=3$. The length scale of streak growth and decay is not affected by wall suction. To compare the streak attenuation at $M=3$ and $M=0.8$, the $F_{w}$ values have been adjusted to obtain the same scaled wall suction velocities $V_{w}$ for the two Mach number cases. These changes are necessary because $V_{w}$ depends on $U_{\infty}^{*}\left(\right.$ refer to (7)). The $F_{w}$ value at $M$
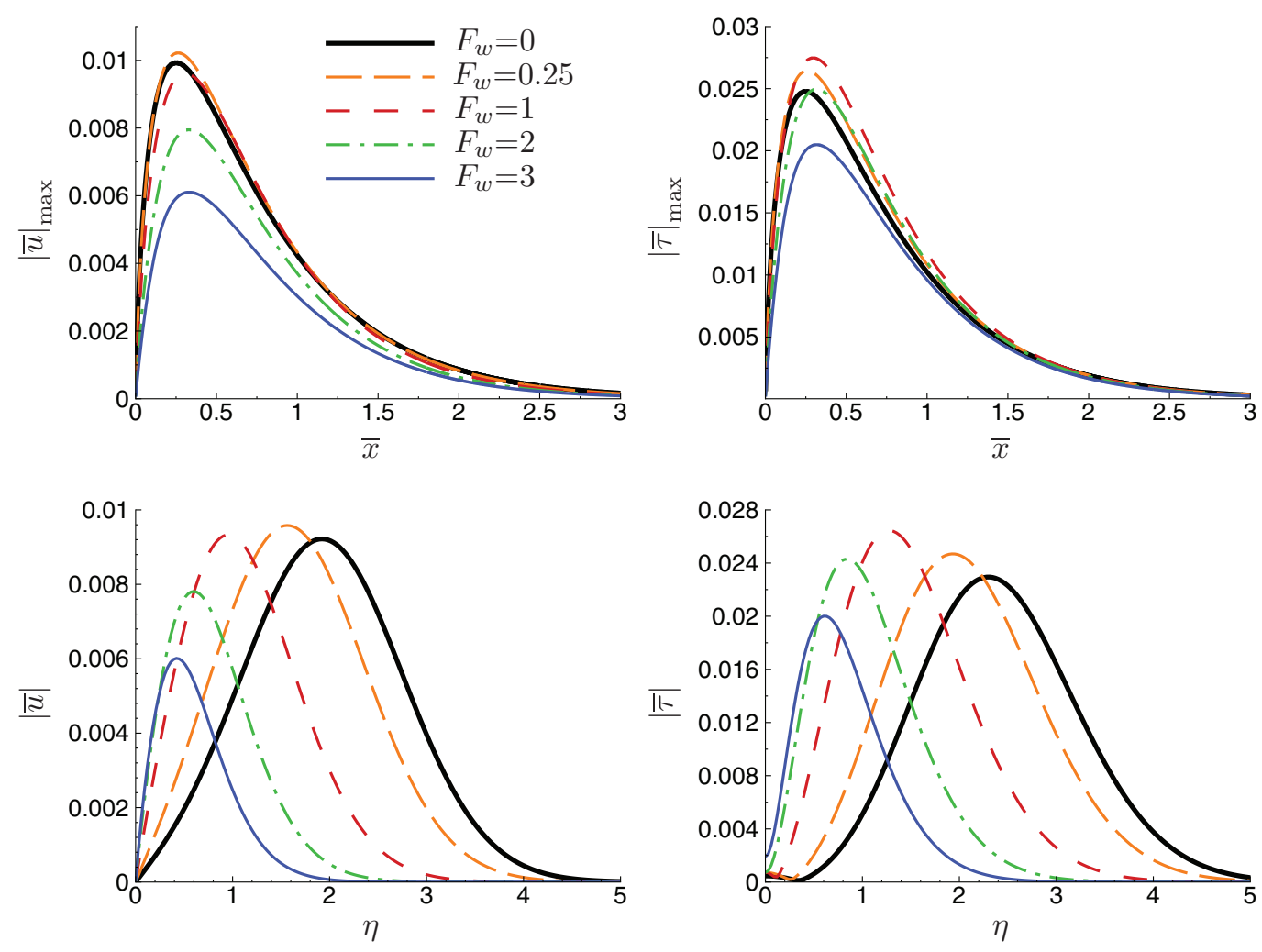

FIG. 5. Top: Maximum values along $\eta$ of streamwise velocity $|\bar{u}|$ (left) and temperature $|\bar{\tau}|$ (right) as functions of $\bar{x}$ for $M=3$ and $\kappa=1$ for different $F_{w}$ (suction cases). Bottom: Streamwise velocity $|\bar{u}|$ (left) and temperature $|\bar{\tau}|$ (right) profiles at $\bar{x}=0.4$ for $M=3$ and $\kappa=1$ for different $F_{w}$ (suction cases). 

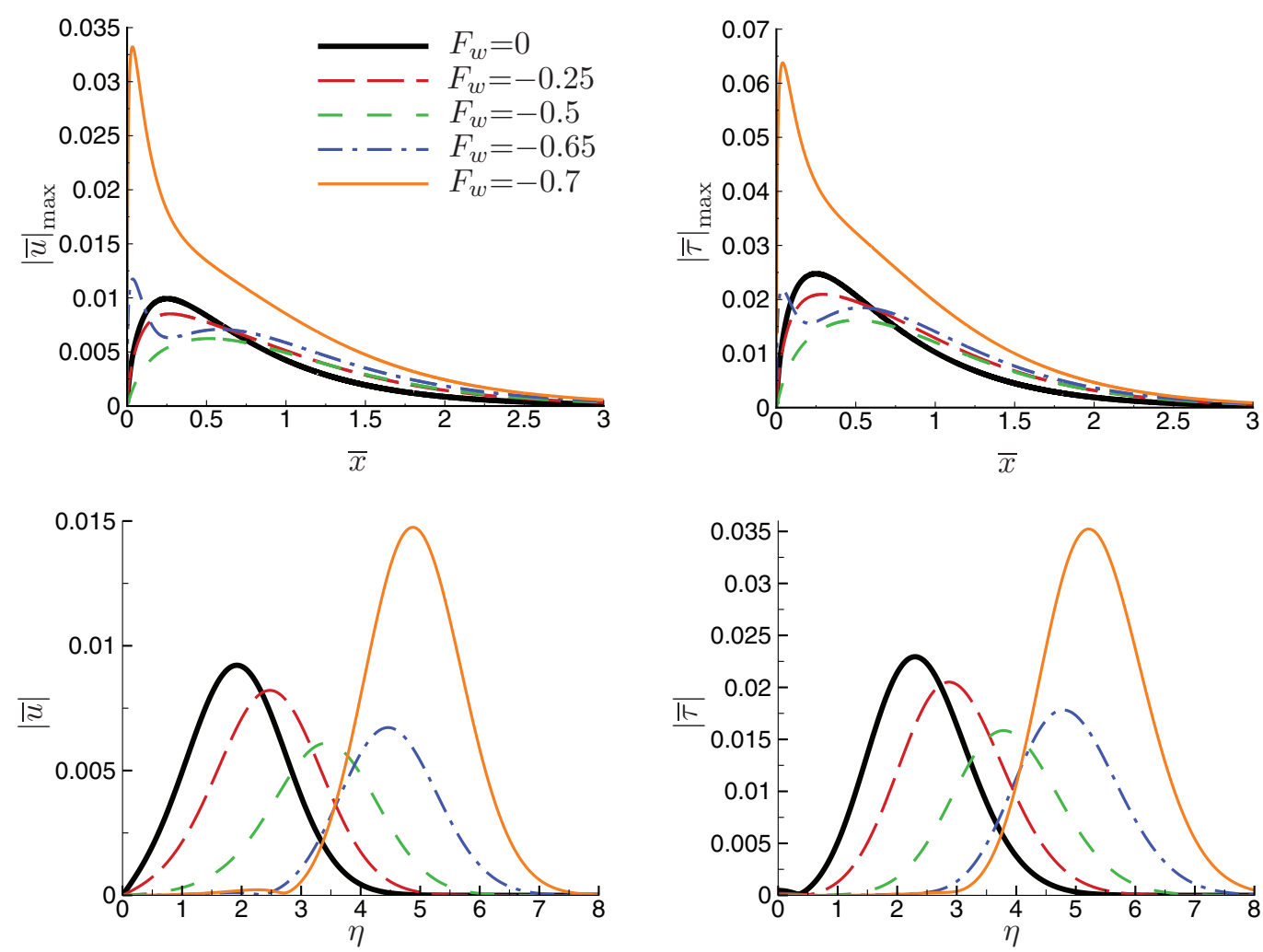

FIG. 6. Top: Maximum values along $\eta$ of streamwise velocity $|\bar{u}|$ (left) and temperature $|\bar{\tau}|$ (right) as functions of $\bar{x}$ for $M=3$ and $\kappa=1$ for different $F_{w}$ (blowing cases). Bottom: Streamwise velocity $|\bar{u}|$ (left) and temperature $|\bar{\tau}|$ (right) profiles at $\bar{x}=0.4$ for $M=3$ and $\kappa=1$ for different $F_{w}$ (blowing cases).

$=0.8$ which gives the same $V_{w}$ value at $M=3$ is thus found as follows: $F_{w}(M=0.8)=F_{w}(M$ $=3)\left[T_{w}(M=3) /\left(T_{w}(M=0.8)\right)\right] \sqrt{0.8 / 3}$, where $v_{\infty}^{*}, \lambda_{z}^{*}$ are assumed constant. The temperature $T_{w}(M=0.8)$ is found iteratively by successive mean-flow computations. In both subsonic and supersonic conditions, the velocity and temperature profiles are brought closer to the wall by suction, the peak at $\bar{x}=0.4$ shifting from $\eta \approx 2$ to $\eta \approx 0.5$ as $F_{w}$ reaches 3 for $M=3$, and 3.8 for $M=0.8$.

The locations of maximum $|\bar{u}|$ and of maximum $|\bar{\tau}|$ along $\eta$ at vanishing small values of $\bar{x}$ are shown in Figure 8 as function of $\delta_{99}$ for the cases shown in Figures 5 and 7. The two quantities are strongly correlated: as the suction intensity increases and the boundary layer becomes thinner, the maximum values decreases almost linearly with $\delta_{99}$. The effect of compressibility on the maximum $\eta$ location is weak. The results at $M=0.8$ agree well with the ones by Ricco and Dilib ${ }^{30}$ for the incompressible case, and the effect of suction is even stronger for $M=0$ than for $M=0.8$ as the peak of the streamwise fluctuations decreases by about a fourth when the suction level reaches $F_{w}=2$.

\section{Klebanoff modes and large-scale streamwise oscillations for $\kappa \lesssim 4 \kappa_{c}$}

In the high-Mach-number subsonic and in supersonic conditions, RW discovered that oblique exponentially growing TS waves may be triggered if the spanwise wavenumber $\kappa$ is lower than a threshold $\kappa_{c}$, which depends mildly on the Mach number. The location of instability shifts downstream as $\kappa$ decreases. In this section, we explore the effects of wall suction on Klebanoff modes which have a wavenumber $\kappa$ which is slightly higher than $\kappa_{c}$, so that no exponential growth is detected. Large-amplitude streamwise oscillations of the streak amplitude occur in the range $\kappa_{c}<\kappa$ $\lesssim 4 \kappa_{c}$ (where the factor 4 is empirically determined), as shown in Figure 9 (left) for the streamwise velocity and in Figure 9 (right) for the temperature for $\kappa=0.05$ and $M=3$. These oscillations 

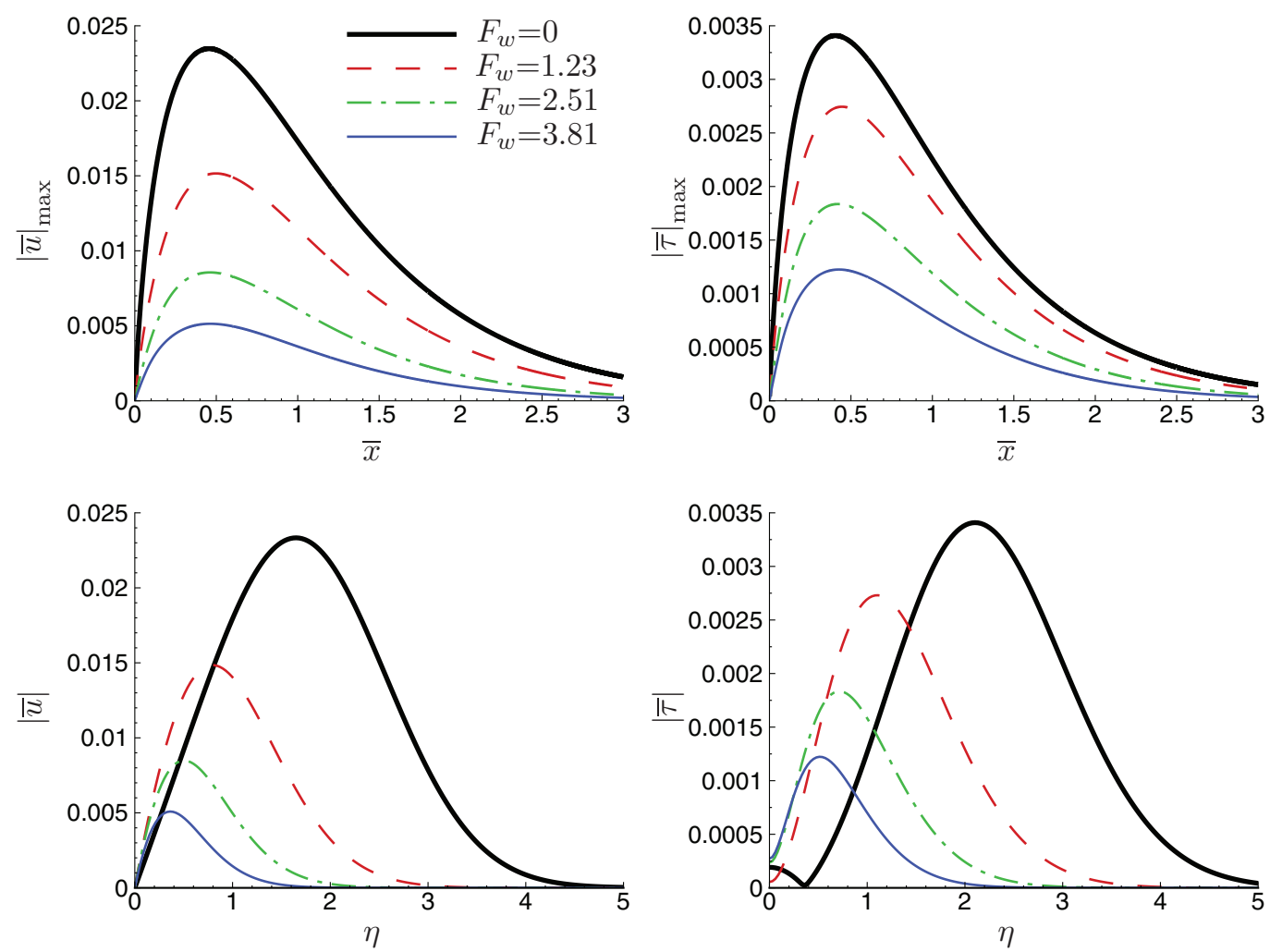

FIG. 7. Top: Maximum values along $\eta$ of streamwise velocity $|\bar{u}|$ (left) and temperature $|\bar{\tau}|$ (right) as functions of $\bar{x}$ for $M$ $=0.8$ and $\kappa=1$. Bottom: Streamwise velocity $|\bar{u}|$ (left) and temperature $|\bar{\tau}|$ (right) profiles at $\bar{x}=0.4$ for $M=0.8$ and $\kappa=1$. The $F_{w}$ values are set so that $V_{w}$ values are equal to the Mach-3 cases in Figure 5.

are of larger amplitude in the supersonic regime than in the subsonic case and occur as soon as the flow develops from the leading edge. They are not observed in the incompressible limit and they were not reported by RW, who focussed only on compressible Klebanoff modes whose dynamics are dominated by viscous effects or are in the very small $\kappa$ limit where TS waves are at work. Their streamwise velocity fluctuations are more intense than the temperature ones. The velocity and temperature profiles are brought closer to the wall by suction (the wall-normal location of the maxima is not shown) and their amplitude is attenuated during the initial evolution, although the streamwise length scale of the modulation is not influenced by the wall transpiration. Further downstream, the streamwise velocity amplitude is not influenced by the wall suction and it only mildly intensifies the amplitude of the temperature fluctuations.

\section{Klebanoff modes and TS waves for $\kappa \lesssim \kappa_{c}$}

This section and Sec. III E present the influence of suction on the low-frequency TS waves discovered by RW in compressible laminar boundary layers for small $\kappa$. This receptivity mechanism operates as follows. The free-stream perturbations trigger quasi three-dimensional Lam-Rott boundary-layer eigensolutions ${ }^{20}$ which develop downstream, together with the Klebanoff modes, but, while the latter are continuously forced by the free-stream gusts and are therefore solutions of an inhomogeneous differential system, the former are homogeneous solutions of the boundary layer equations. The intensity of the Lam-Rott modes drops and their wavelength shortens as they evolve downstream. Goldstein ${ }^{14}$ first discovered that these fluctuations, previously regarded as innocuous in the transition process because of the exponential decay, are instead crucial for instability. For highfrequency acoustic free-stream perturbations, these modes generate a streamwise pressure gradient which, once comparable with the inertial forces in a very thin near-wall layer, induces the decaying 


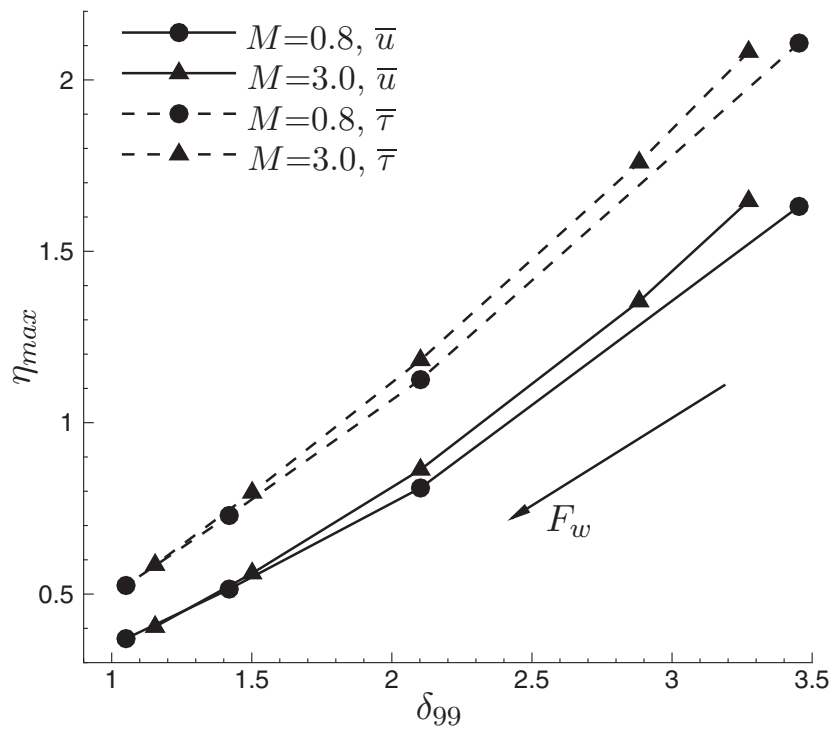

FIG. 8. Wall-normal $\eta$ locations of maximum $|\bar{u}|$ and of maximum $|\bar{\tau}|$ for $\bar{x} \rightarrow 0$ as function of $\delta_{99}$ for the cases in Figures 5 and 7.

modes to become unstable TS waves. RW have shown that a spanwise pressure gradient is instead engendered when the free-stream perturbations are of low-frequency, such as the convected gusts. The spanwise pressure gradient interferes with the viscous flow by creating a spanwise velocity component which, once of the same order of magnitude of the streamwise and wall-normal velocity components, causes an oblique TS wave to grow downstream. The starting location of growth can be quite close to the leading edge for Mach numbers higher than 0.8 , whereas, in the incompressible limit, it moves downstream significantly and this mechanism is therefore not at work in practise. More details on the mathematical and physical analysis are found in RW.

In this section, the perturbations are studied for $\kappa$ smaller than $\kappa_{c}$. As the $\kappa$ value is chosen to be comparable with $\kappa_{c}$, the growth is observed in the proximity of the leading edge. Figure 10 shows the profiles of streamwise, wall-normal, spanwise, and temperature perturbations at $\bar{x}=15$ for $M=3$ and $\kappa=0.02$ (the threshold values at this Mach number is $\kappa_{c} \approx 0.03$ ). The wall suction damps the velocity components within the boundary layer and the gradients near the wall are significantly reduced for all three components. The temperature fluctuations are instead intensified at this $\bar{x}$. At
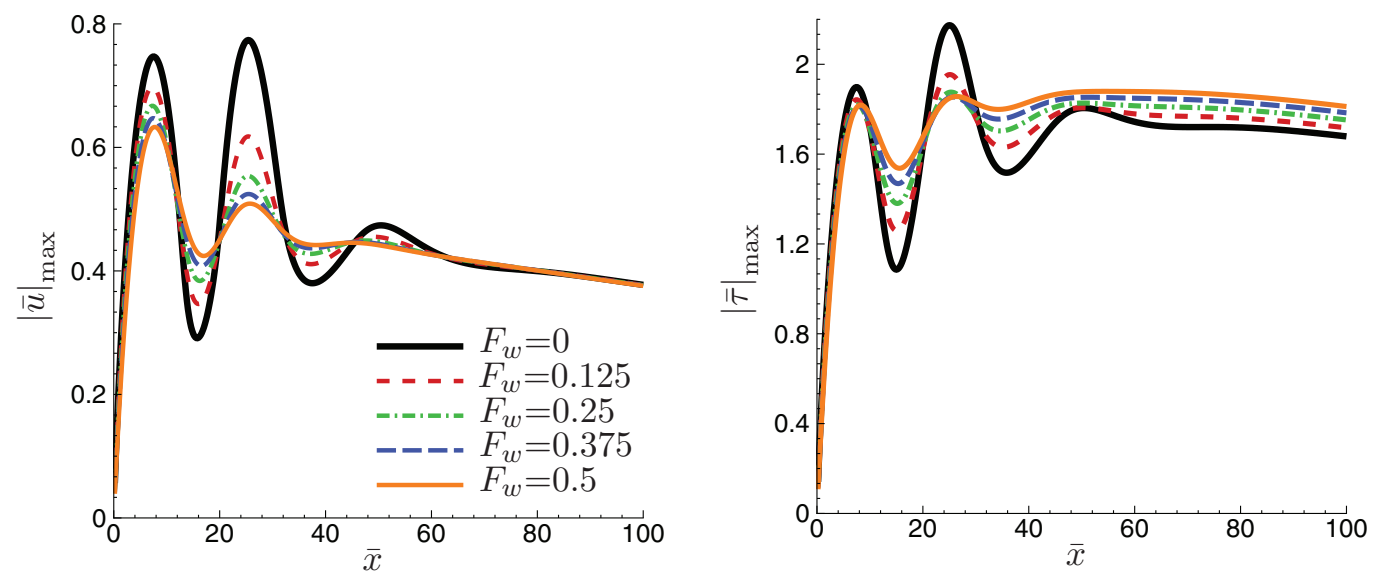

FIG. 9. Streamwise evolution of maximum streamwise velocity $|\bar{u}|_{\max }$ (left) and maximum temperature $|\bar{\tau}|_{\max }$ (right) for $M=3$ and $\kappa=0.05$. 

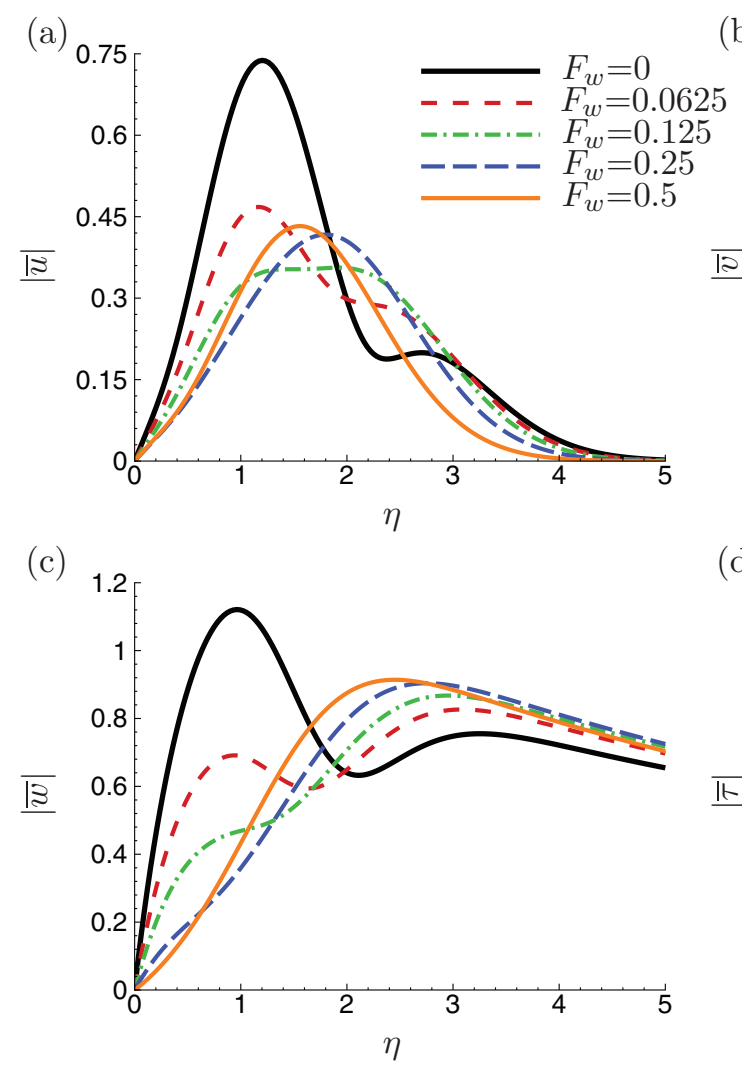

(b)

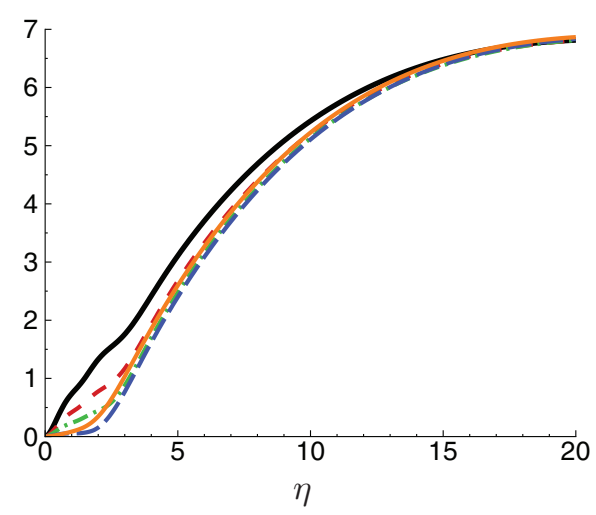

(d)

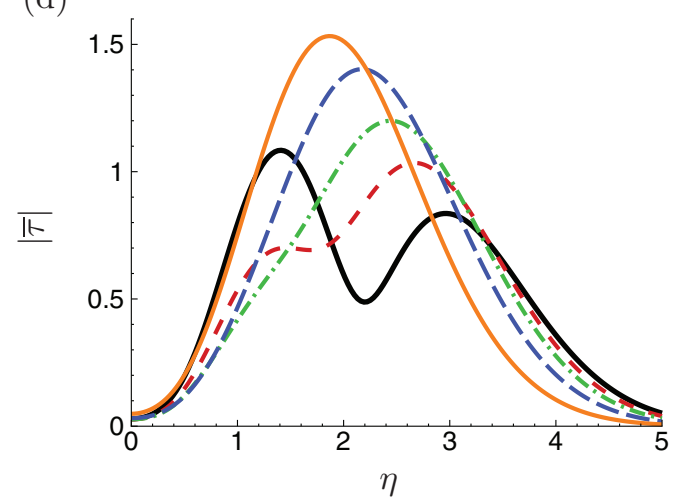

FIG. 10. Wall-normal profiles of (a) streamwise velocity $|\bar{u}|$, (b) wall-normal velocity $|\bar{v}|$, (c) spanwise velocity $|\bar{w}|$, and (d) temperature $|\bar{\tau}|$ for $M=3, \kappa=0.02$ at $\bar{x}=15$ for different suction levels.

this downstream location, the exponential growth has commenced, as shown in Figure 11. Wall suction retards the onset of instability of both the streamwise velocity (left graph) and temperature (right graph) fluctuations. The effect of suction is slightly more effective on the velocity than on the temperature.

In Figure 12, the absolute value and the real part of $\bar{u}$ are shown as a function of $\bar{x}$ for their entire downstream evolution and different suction intensities. The waves undergo an intense amplification, reach a peak, and eventually decay at almost the same rate of the growth. Once established, the waves possess a well-defined streamwise wavelength. The disturbance amplitude is reduced by an order
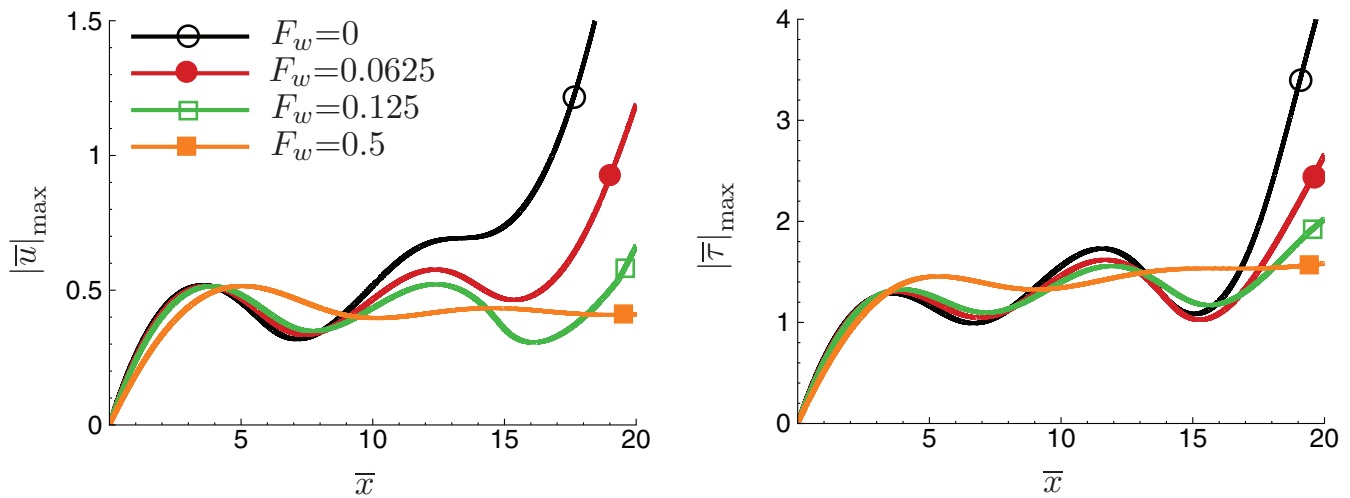

FIG. 11. Streamwise evolution of maximum streamwise velocity $|\bar{u}|_{\max }$ (left) and maximum temperature $|\bar{\tau}|_{\max }$ (right) for $M=3$ and $\kappa=0.02$. 

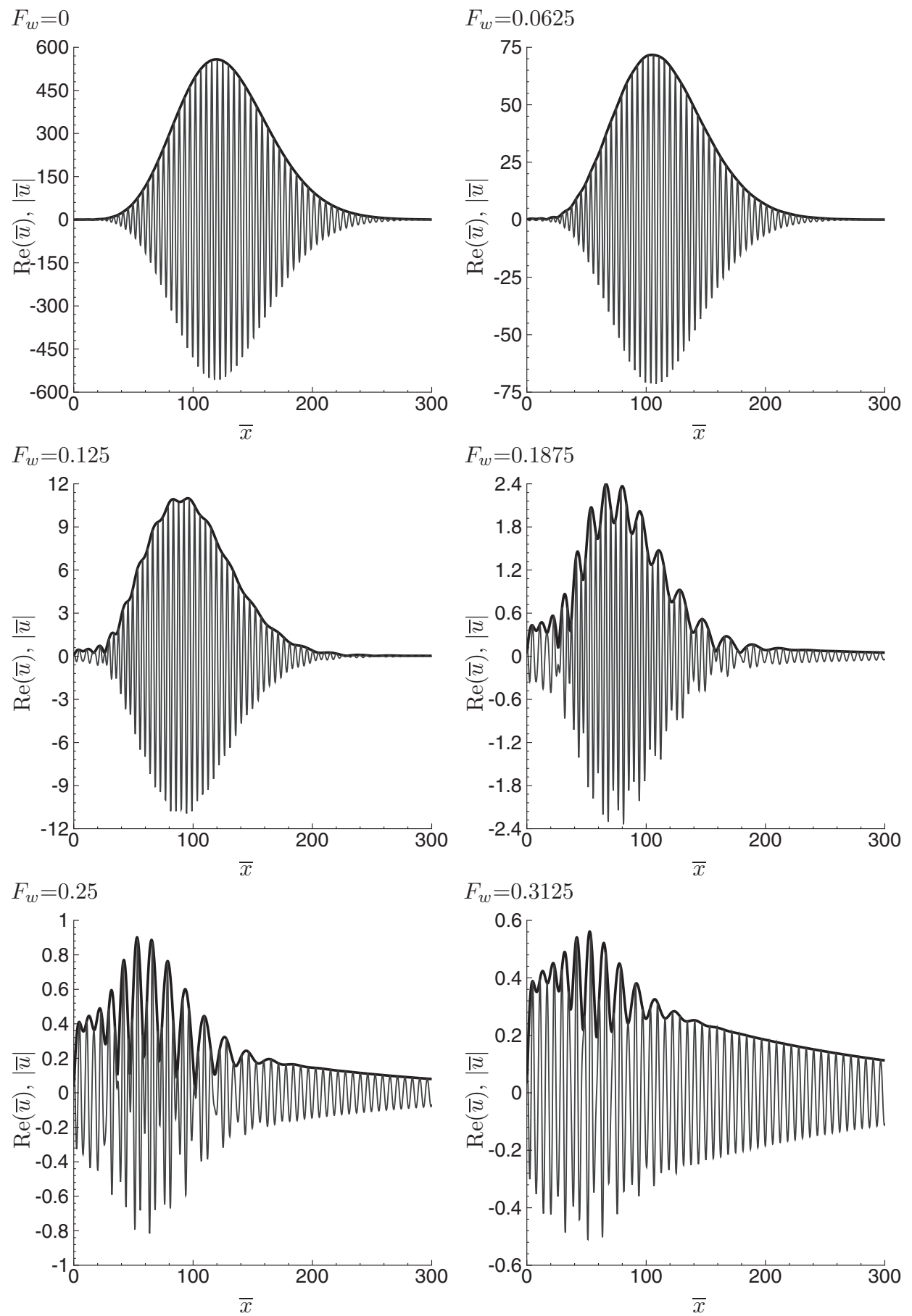

FIG. 12. Downstream evolution of $\operatorname{Re}(\bar{u})$ and $|\bar{u}|$ of growing disturbance for $M=3, \kappa=0.02$ and different suction levels.

of magnitude for wall suction as low as $F_{w}=0.0625$, and by two orders of magnitude by further increasing the transpiration level to $F_{w}=0.1875$. For $F_{w}=0.125$ and above, the envelope of the wave shows streamwise modulations whose wavelength is about three times the original wavelength. In the range $0.125 \leq F_{w} \leq 0.25$, the amplitude of this superposed oscillation remains approximately constant, and it is only until suction has increased to $F_{w}=0.3125$ that it also experiences attenuation. The TS waves are fully attenuated for $F_{w}>0.5$. 

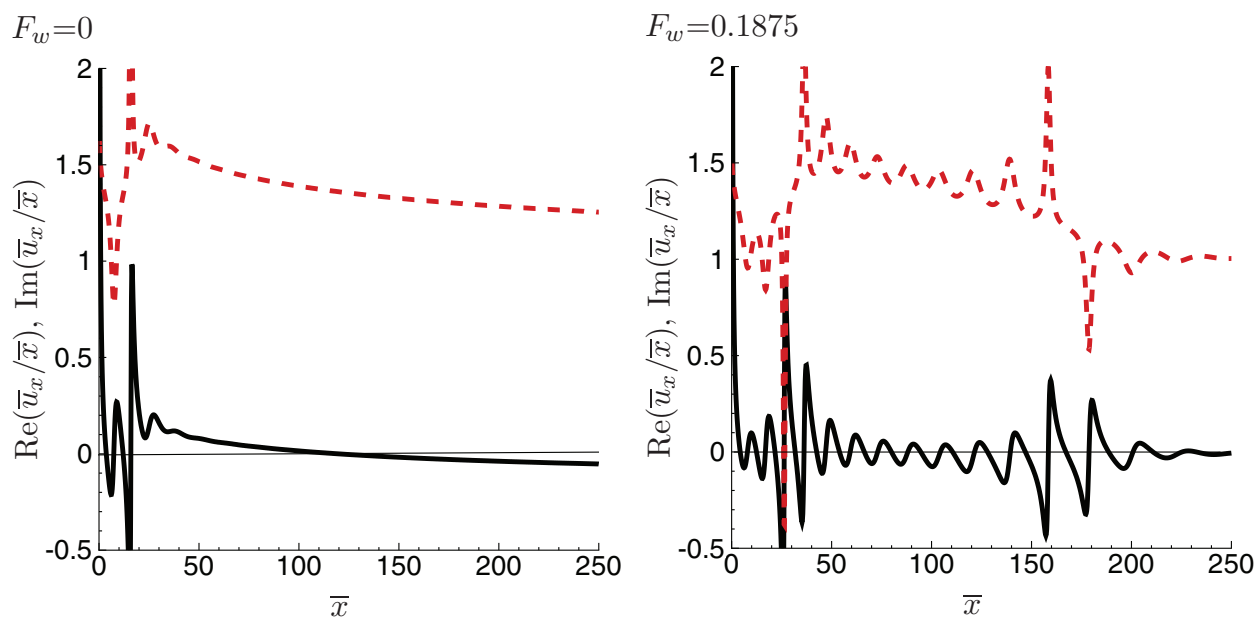

FIG. 13. The real (growth rate - solid lines) and imaginary (wavenumber - dashed line) parts of the ratio $\bar{u}_{x} / \bar{u}$ as functions of $\bar{x}$ for $M=3, \kappa=0.02$ in the no-transpiration case (left) and for $F_{w}=0.1875$ (right).

The growth rate and wavenumber can be extracted from the numerical calculations as the real and the imaginary parts of $\bar{u}_{x} / \bar{u}$, respectively, where the subscript $x$ indicates the partial derivative with respect to $\bar{x}$. The growth rate and wavenumbers are shown in Figure 13 for the no-transpiration case (left) and for $F_{w}=0.1875$ (right). The wavenumber is hardly affected by wall suction.

\section{E. Klebanoff modes and TS waves for $\kappa \ll 1$}

In this section, the disturbance dynamics are studied for very small $\kappa$ values. The downstream evolution of streamwise velocity and temperature is shown in Figure 14 for $M=3, \kappa=0.001$, and $F_{w}=3$. Downstream of the initial growth, the kinematic and thermal streaks persist and are not attenuated by viscous effects. After reaching their peak, the velocity disturbances are only mildly attenuated downstream. The thermal streaks initially grow at the same rate of the kinematic streaks, and then more gradually after the velocity disturbances have reached their maximum. Wall suction initially causes the disturbances to grow more slowly, but then has an amplifying effect further downstream.

The wall-normal profiles of the velocity and temperature disturbances are shown in Figures 15 and 16. In the no-transpiration case, the disturbances move towards the outer part of the boundary layer as they develop downstream, i.e., they confine themselves in the edge layer (refer to LWG for a detailed account on this process). In the wall suction case, the edge-layer phenomenon is strongly
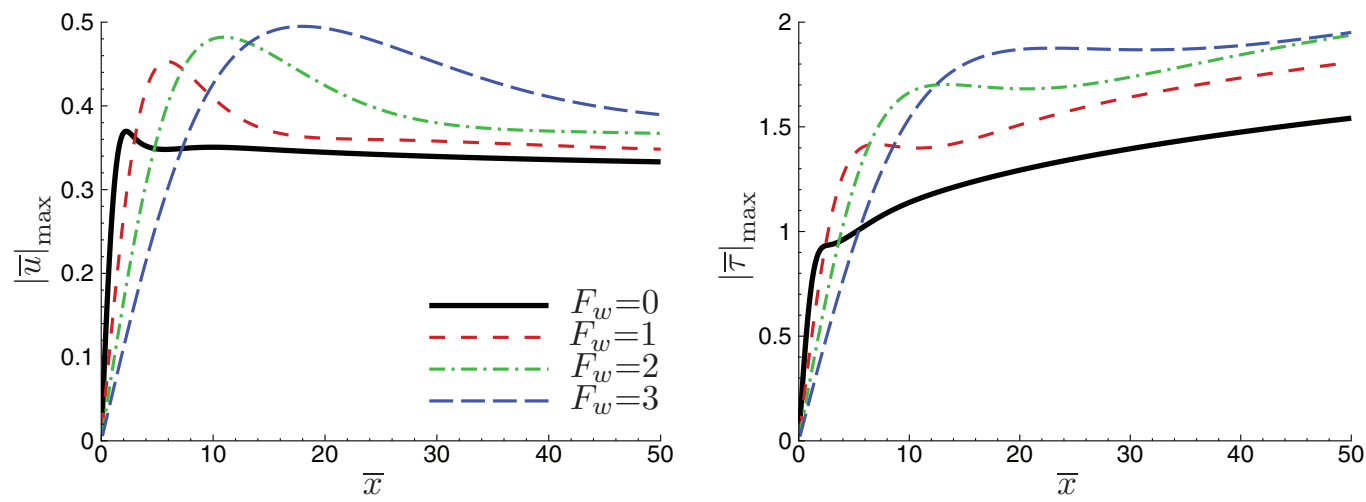

FIG. 14. Peak disturbance amplitudes of streamwise velocity $\bar{u}$ (left) and temperature (right) for $\kappa=0.001$ and $M=3$. 

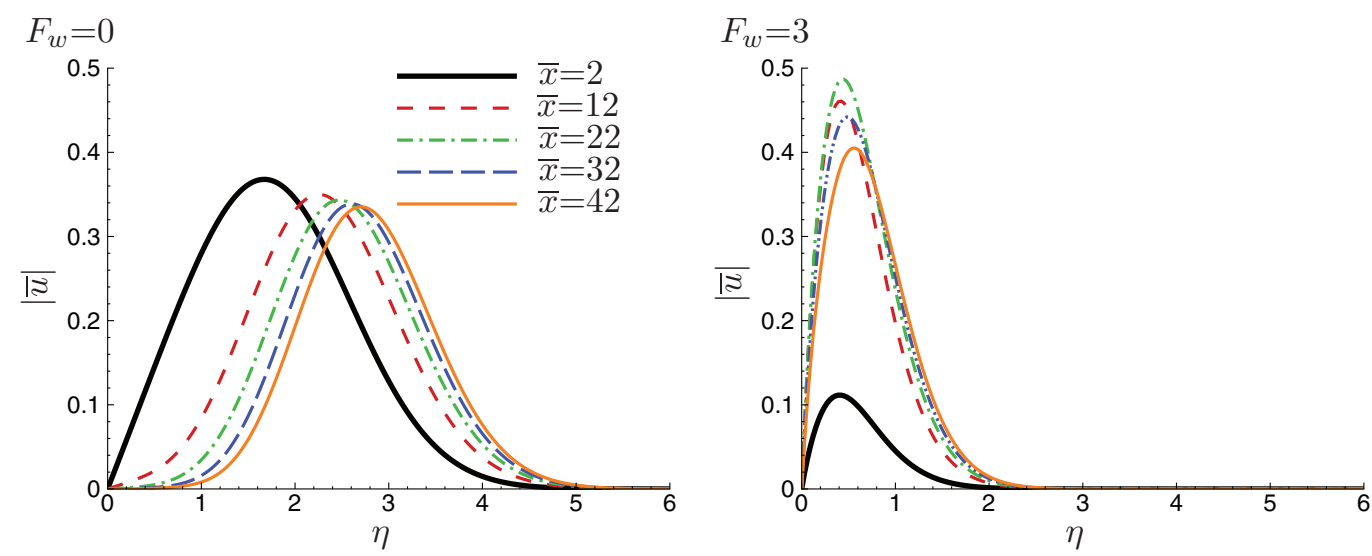

FIG. 15. Profiles of streamwise velocity for $\kappa=0.001$ and $M=3$ at different downstream locations in the no-transpiration case (left) and with suction, $F_{w}=3$ (right).

attenuated and the perturbations are brought closer to the wall. The temperature fluctuations at the wall are enhanced for wall adiabatic conditions as the boundary layer becomes thinner.

Wall suction has a dramatic stabilizing effect on the TS-wave instability, which occurs far downstream when $\kappa \ll 1$. Figure 17 (top) shows the growth rates and the locations for the onset of TS waves at $M=3$ for $\kappa=0.0005$ and different suction levels. The onset of exponential growth shifts downstream as $F_{w}$ increases. The wavenumbers, shown in Figure 17 (bottom), exhibit only mild changes with wall suction. The growth rate and the wavenumber may also be determined by triple-deck asymptotic analysis. The interested reader should refer to RW for further details. As the instability location $\bar{x}_{c}$ occurs for $\kappa \ll 1, \bar{x} \gg 1$, a new streamwise coordinate is defined as $x_{1}=\kappa \bar{x}$ $=\mathcal{O}(1)$. The streamwise velocity component is assumed to grow as follows:

$$
\bar{u} \sim \exp \left[\frac{\mathrm{i}}{\kappa^{1 / 2}} \int_{0}^{\bar{x}} \alpha_{1}\left(x_{1}\right) \mathrm{d} \breve{x}_{1}\right] .
$$

The growth rate and the wavenumber are extracted as $-\operatorname{Im}\left(\alpha_{1}\right) / \kappa^{1 / 2}$ and $\operatorname{Re}\left(\alpha_{1}\right) / \kappa^{1 / 2}$, respectively. The complex wavenumber $\alpha_{1}=\alpha_{1}\left(x_{1}\right)$ is found through the dispersion relation ${ }^{35,39}$

$$
\Delta\left(x_{1}, \alpha_{1}\right) \equiv \int_{\eta_{0}}^{\infty} \operatorname{Ai}(\check{\eta}) \mathrm{d} \check{\eta}-\frac{\mu(0)^{1 / 3}}{T(0)^{7 / 3}}\left(\frac{F^{\prime \prime}(0)}{\sqrt{2 x_{1}}}\right)^{5 / 3}\left(\mathrm{i}_{1}\right)^{-1 / 3} \operatorname{Ai}^{\prime}\left(\eta_{0}\right)=0,
$$
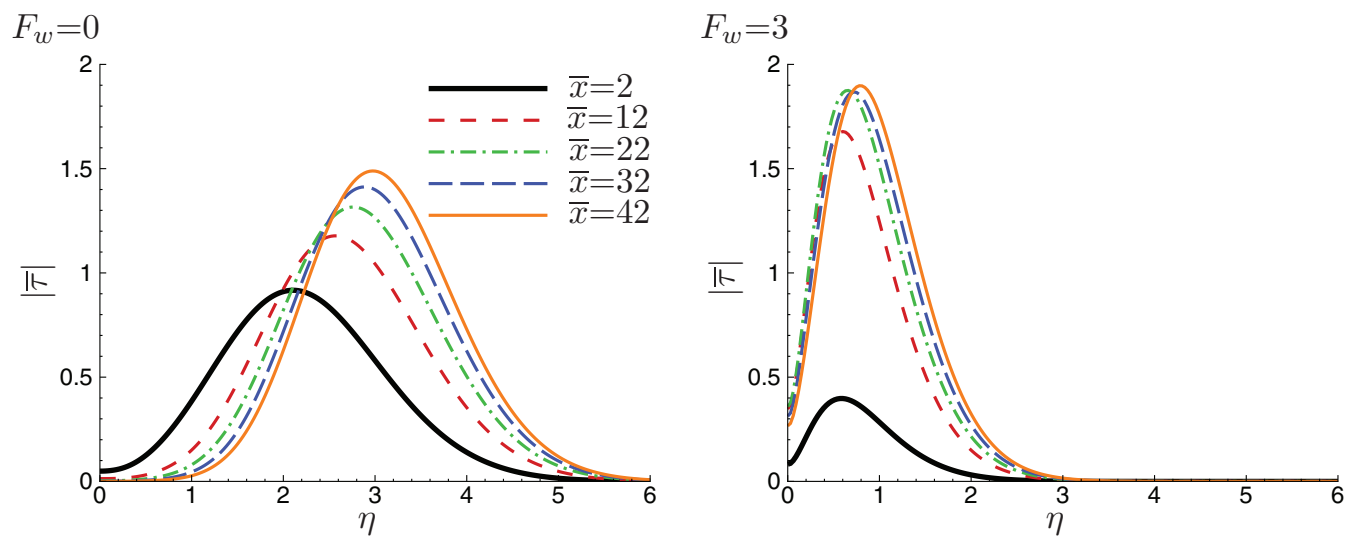

FIG. 16. Profiles of temperature for $\kappa=0.001$ and $M=3$ at different downstream locations in the no-transpiration case (left) and with suction, $F_{w}=3$ (right). 

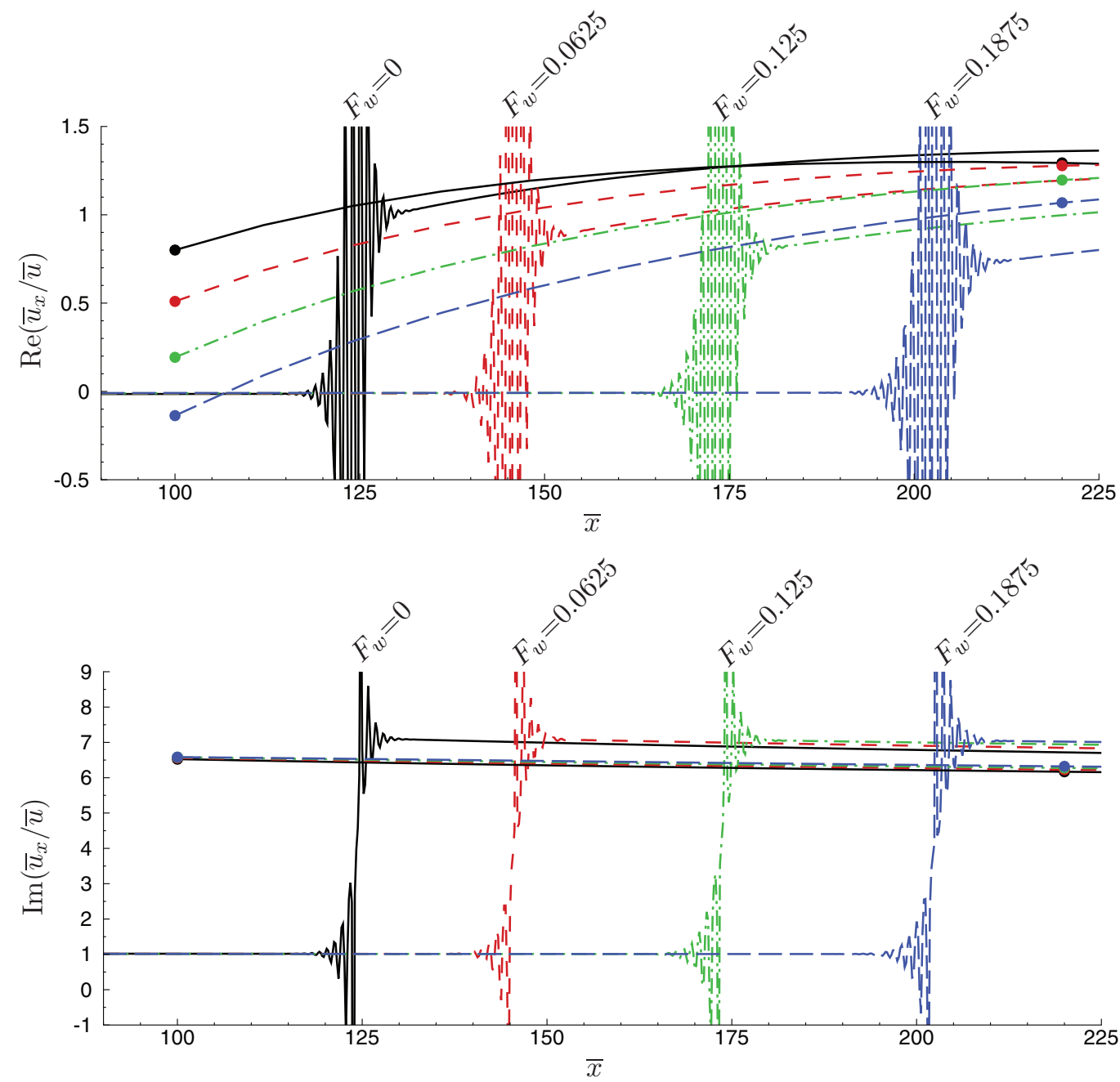

FIG. 17. Downstream evolution of growth rate (top) and wavenumber (bottom) of growing disturbance with $\kappa=0.0005$ and $M=3$ for different suction levels. The curved marked by the dots are computed via the triple-deck dispersion relation (20).

where Ai indicates the Airy function of the first kind, the prime denotes its first derivative, and

$$
\eta_{0}=-\left(\alpha_{1} F^{\prime \prime}(0)\right)^{-1}\left(2 \mathrm{i} F^{\prime \prime}(0) \alpha_{1} x_{1} T(0) / \mu(0)\right)^{1 / 3} .
$$

The effects of compressibility and wall suction are distilled in (20) through the wall quantities $\mu(0)$, $T(0)$, and $F^{\prime \prime}(0)$. The numerically computed growth rates and wavenumbers are compared with their triple-deck counterparts in Figure 17. The wavenumbers are computed via triple-deck theory more accurately than the growth rates. The downstream shift of instability is captured by the theory and even the small changes of wavenumber with wall suction are predicted. For both quantities, the agreement worsens as the suction intensity increases.

\section{SUMMARY}

The dynamics of unsteady streaks or Klebanoff modes in laminar boundary layers is a steadily growing subject in the fluid mechanics research community as these structures are thought to be precursors to transition in flows with moderate to high levels of free-stream turbulence. ${ }^{25,31}$ These streaks have been studied quite extensively in the incompressible regime, while works on the effects of compressibility are more limited. The compressible Klebanoff modes have been first investigated 
by RW by extending the seminal study by LWG, which focussed on the incompressible limit. RW's mathematical framework has been used to investigate the influence of steady mean-flow wall suction on the growth of compressible streaks in a laminar boundary layer.

The Klebanoff perturbations are studied for different regimes distinguished by $\kappa$, the scaled spanwise wavenumber of the perturbation, at a distance from the leading edge which is comparable with the streak streamwise length scale. At these downstream locations, $\kappa$ is a measure of the ratio between $\delta_{99}^{*}$, the mean-flow boundary layer thickness, and $\lambda_{z}^{*}$, the streak spanwise wavelength. We can therefore list the results as follows:

- $\delta_{99}^{*} \gg \lambda_{z}^{*}$ : In this asymptotic limit, $\kappa \gg 1$, the numerical solutions can be rescaled conveniently and satisfy the steady compressible boundary region equations. The effect of suction is moderate on the streamwise velocity fluctuations and negligible on the temperature fluctuations in the limit of very small spanwise wavelengths.

- $\delta_{99}^{*} \sim \lambda_{z}^{*}$ : When $\kappa=\mathcal{O}(1)$ and in supersonic conditions, boundary-layer temperature fluctuations can be amplified more significantly with respect to the free-stream value than the velocity fluctuations. Small suction intensities can enhance the fluctuations in the supersonic regime, while high suction levels are always stabilizing at all Mach numbers.

- $\delta_{99}^{*} \lesssim \lambda_{z}^{*}$ : This indicates a flow where $\kappa$ is smaller than unity, but not as small as to enter the receptivity regime for which oblique TS waves are triggered, usually for $\kappa=0.02-0.03$ in supersonic conditions. For the first time, large streamwise oscillations are found to occur as the result of a competing effect between the unstable growth of the TS waves and the stabilizing effect of viscosity. Viscous effects therefore have two roles in this regime: they promote the growth of instability waves through the action of the wall-shear stress $F^{\prime \prime}(0)$ (refer to the dispersion relation (20)), but they also tend to suppress the disturbance growth through the viscous diffusion terms in (15)-(17). These oscillations are more intense in the supersonic regime than in the subsonic regime and are not observed in the incompressible case. A small level of suction can effectively damp their amplitude.

- $\delta_{99}^{*} \ll \lambda_{z}^{*}$ : For $\kappa \ll 1$ suction attenuates the Klebanoff modes near the leading edge and amplifies them further downstream. For certain wavenumbers, TS waves dominate the flow for high Mach number subsonic conditions and in the supersonic regime. Suction has a dramatic effect on these waves. Their intensity can be diminished by an order of magnitude by very small levels of suction and the location at which they are triggered shifts downstream significantly. The range of $\kappa$ values for which TS are observed becomes smaller. As the level of suction increases, the fundamental wavelength of these modes is largely unaffected while a superposed streamwise modulation is found whose amplitude is not influenced by the suction level. Tripledeck theory predicts the intense changes in growth rate and the very mild modifications in wavenumber.

In brief, we conclude that wall suction is more effective at attenuating the streak intensity in the incompressible limit and in the subsonic case than in the supersonic cases, i.e., the effectiveness of wall suction deteriorates as the Mach number increases. The next step is to verify our results via direct numerical simulations to simulate the entire transition process, for example, using the method by Pirozzoli and Grasso ${ }^{28}$ and Pirozzoli et al. ${ }^{27}$ and to assess the effectiveness of wall suction to delay transition. Further motivation arises from the small values of actual suction velocity needed to obtain a significant suppression of compressible Klebanoff modes or TS waves. For an aircraft cruising at $U_{\infty}^{*}=265 \mathrm{~m} \mathrm{~s}^{-1}$, a wall suction of strength $F_{w}=1$ corresponds to $V_{w}^{*}=-0.11 \mathrm{~m} \mathrm{~s}^{-1}$. This gives a dimensional suction velocity of just $0.05 \%$ of the free-stream velocity, indicating that wall transpiration is a potentially practical method for reducing drag on a cruising aircraft.

\section{ACKNOWLEDGMENTS}

EPSRC First Grant EP/I033173/1 partly supported P.R. and fully supported P.D.H.

\footnotetext{
${ }^{1}$ P. Andersson, M. Berggren, and D. S. Henningson, "Optimal disturbances and bypass transition in boundary layers," Phys.
} Fluids 11(1), 134-150 (1999). 
${ }^{2}$ P. Balakumar and P. Hall, "On the control of the fastest growing Gortler vortex," Theor. Comp. Fluid Dyn. 13, 21-31 (1999).

${ }^{3}$ P. Balakumar and P. Hall, "Optimum suction distribution for transition control," Theor. Comp. Fluid Dyn. 13, 1-19 (1999).

${ }^{4}$ R. J. Bodonyi and P. W. Duck, "Boundary-layer receptivity due to a wall suction and control of Tollmien-Schlichting waves," Phys. Fluids 4(6), 1206-1214 (1992).

${ }^{5}$ A. L. Braslow, A History of Suction-Type Laminar-Flow Control with Emphasis on Flight Research, Monograph in Aerospace History No. 13 (NASA Historic Division, 1999).

${ }^{6}$ M. G. Bystrom, O. Levin, and D. S. Henningson, “Optimal disturbances in suction boundary layers," Eur. J. Mech. B/Fluids 26(3), 330-343 (2007).

${ }^{7}$ T. Cebeci, Convective Heat Transfer (Springer-Verlag, 2002).

${ }^{8} \mathrm{H}$. Deyhle and H. Bippes, "Disturbance growth in an unstable three-dimensional boundary layer and its dependence on environmental conditions," J. Fluid Mech. 316, 73-113 (1996).

${ }^{9}$ H. L. Dryden, "Air flow in the boundary layer near a plate," NACA Report No. 562, 1936.

${ }^{10}$ H. L. Dryden, "Transition from laminar to turbulent flow at subsonic and supersonic speeds," Proceedings of the Conference on High-Speed Aeronautics (Polytechnic of Brooklyn, New York, 1955), Vol. 41.

${ }^{11}$ J. H. M. Fransson and P. Corbett, "Optimal linear growth in the asymptotic suction boundary layer," Eur. J. Mech. B/Fluids 22, 259-270 (2003).

${ }^{12}$ J. H. M. Fransson and P. H. Alfredsson, "On the disturbance growth in an asymptotic suction boundary layer," J. Fluid Mech. 482, 51-90 (2003).

${ }^{13}$ M. Gad-el Hak, Flow Control - Passive, Active and Reactive Flow Management (Cambridge University Press, 2000).

${ }^{14}$ M. E. Goldstein, "The evolution of Tollmien-Schlichting waves near a leading edge," J. Fluid Mech. 127, 59-81 (1983).

${ }^{15}$ A. N. Gulyaev, V. E. Kozlov, V. R. Kuzenetsov, B. I. Mineev, and A. N. Sekundov, "Interaction of a laminar boundary layer with external turbulence," Fluid Dyn. 24(5), 700-710 (1989) [Izv. Akad. Nauk. SSSR, Mekh. Zhidk. Gaza 5, 55-65 (1989)].

${ }^{16}$ C. Gustavsson, "Development of three-dimensional disturbances in boundary layers with suction," Master's thesis (Luleå University of Technology, 2000).

${ }^{17}$ R. D. Joslin, “Aircraft laminar flow control,” Ann. Rev. Fluid Mech. 30, 1-29 (1998).

${ }^{18}$ J. M. Kendall, "Studies on laminar boundary layer receptivity to free-stream turbulence near a leading edge," in Boundary Layer Stability and Transition to Turbulence, edited by D. C. Reda, H. L. Reed, and R. Kobayashi (ASME FED, 1991), Vol. 114, pp. 23-30.

${ }^{19}$ P. S. Klebanoff, "Effect of free-stream turbulence on a laminar boundary layer," Bull. Am. Phys. Soc. 10(11), 1323 (1971).

${ }^{20}$ S. H. Lam and N. Rott, "Eigen-functions of linearized unsteady boundary layer equations," J. Fluids Eng. 115, 597-602 (1993).

${ }^{21}$ S. J. Leib, D. W. Wundrow, and M. E. Goldstein, "Effect of free-stream turbulence and other vortical disturbances on a laminar boundary layer," J. Fluid Mech. 380, 169-203 (1999).

${ }^{22}$ O. Levin, E. N. Davidsson, and D. S. Henningson, "Transition thresholds in the asymptotic suction boundary layer," Phys. Fluids 17, 114104 (2005).

${ }^{23}$ H. G. Lew and J. B. Fanucci, "On the laminar compressible boundary layer over a flat plate with suction and injection," J. Aeronaut. Sci. 22(9), 589-597 (1955).

${ }^{24}$ P. Luchini, "Reynolds-number-independent instability of the boundary layer over a flat surface: optimal perturbations," J. Fluid Mech. 404, 289-309 (2000).

${ }^{25}$ M. Matsubara and P. H. Alfredsson, "Disturbance growth in boundary layers subjected to free-stream turbulence, J J. Fluid Mech. 430, 149-168 (2001).

${ }^{26}$ S. R. Pate, "Supersonic boundary-layer transition: effects of roughness and free-stream disturbances," AIAA J. 9, 797-803 (1971).

${ }^{27}$ S. Pirozzoli, M. Bernardini, and F. Grasso, "On the dynamical relevance of coherent vortical structures in turbulent boundary layers," J. Fluid Mech. 648, 325-349 (2010).

${ }^{28}$ S. Pirozzoli and F. Grasso, "Direct numerical simulation of impinging shock wave/turbulent boundary layer interaction at $\mathrm{M}=2.25$," Phys. Fluids 18, 065113 (2006).

${ }^{29} \mathrm{P}$. Ricco, "The pre-transitional Klebanoff modes and other boundary layer disturbances induced by small-wavelength free-stream vorticity,” J. Fluid Mech. 638, 267-303 (2009).

${ }^{30} \mathrm{P}$. Ricco and F. Dilib, "The influence of wall suction and blowing on laminar boundary-layer streaks generated by free-stream vortical disturbances," Phys. Fluids 22, 044101 (2010).

${ }^{31} \mathrm{P}$. Ricco, J. Luo, and X. Wu, "Evolution and instability of unsteady nonlinear streaks generated by free-stream vortical disturbances," J. Fluid Mech. 677, 1-38 (2011).

${ }^{32}$ P. Ricco, D.-L. Tran, and G. Ye, "Wall heat transfer effects on Klebanoff modes and Tollmien-Schlichting waves in a compressible boundary layer,” Phys. Fluids 21(2), 024106 (2009).

${ }^{33} \mathrm{P}$. Ricco and $\mathrm{X}$. Wu, "Response of a compressible laminar boundary layer to free-stream vortical disturbances," J. Fluid Mech. 587, 97-138 (2007).

${ }^{34} \mathrm{H}$. Schlichting and K. Gersten, Boundary-Layer Theory (Springer, 2000).

${ }^{35}$ F. T. Smith, "On the first-mode instability in subsonic, supersonic or hypersonic boundary layers," J. Fluid Mech. 198, 127-153 (1989).

${ }^{36}$ K. Stewartson, The Theory of Laminar Boundary Layers in Compressible Fluids (Claredon Press, Oxford, 1964).

${ }^{37}$ G. I. Taylor, "Some recent developments in the study of turbulence," in Fifth International Congress for Applied Mechanics, edited by J. P. Den Hartog and H. Peters (Wiley, New York, 1939), pp. 294-310. 
${ }^{38}$ K. J. A. Westin, A. V. Boiko, B. G. B. Klingmann, V. V. Kozlov, and P. H. Alfredsson, "Experiments in a boundary layer subjected to free stream turbulence. Part 1. Boundary layer structure and receptivity," J. Fluid Mech. 281, 193-218 (1994).

${ }^{39} \mathrm{X}$. Wu, "Generation of Tollmien-Schlichting waves by convecting gusts interacting with sound," J. Fluid Mech. 397, 285-316 (1999).

${ }^{40} \mathrm{X}$. Wu and M. Choudhari, "Linear and non-linear instabilities of a Blasius boundary layer perturbed by streamwise vortices. Part 2. Intermittent instability induced by long-wavelength Klebanoff modes," J. Fluid Mech. 483, 249-286 (2003).

${ }^{41}$ D. W. Wundrow and M. E. Goldstein, "Effect on a laminar boundary layer of small-amplitude streamwise vorticity in the upstream flow," J. Fluid Mech. 426, 229-262 (2001).

${ }^{42}$ S. Yoshioka, J. H. M. Fransson, and P. H. Alfredsson, "Free stream turbulence induced disturbances in boundary layers with wall suction," Phys. Fluids 16(10), 3530-3539 (2004).

${ }^{43}$ S. Zuccher, P. Luchini, and A. Bottaro, "Algebraic growth in a Blasius boundary layer: optimal and robust control by mean suction in the nonlinear regime," J. Fluid Mech. 513, 135-160 (2004). 